\title{
TITLE:
}

\section{Some extensions of the Siegel-Weil formula}

$\operatorname{AUTHOR}(S):$

KUDLA, STEPHEN S.

CITATION:

KUDLA, STEPHEN S.. Some extensions of the Siegel-Weil formula. 数理 解析研究所講究録 1992, 805: 51-87

ISSUE DATE:

1992-08

URL:

http://hdl.handle.net/2433/82931

RIGHT: 


\section{Some extensions of the Siegel-Weil formula \\ Stephen S. Kudla}

\section{Introduction.}

In this article I will survey some relatively recent joint work with S.Rallis, in which we extend the classical formula of Siegel and Weil. In the classical case, this formula identifies a special value of a certain Eisenstein series as an integral of a theta function. Our extension identifies the residues of the (normalized) Eisenstein series on $S p(n)$ as 'regularized' integrals of theta functions. Moreover, we obtain an analogous result for the value of the Eisenstein series at its center of symmetry (when $n$ is odd). Both of these identities have applications to special values and poles of Langlands $L$-functions. Most of our results were announced by Rallis in his lecture [36] at the ICM in Kyoto in August of 1990, and detailed proofs will appear shortly [23]. Thus the present article will be mostly expository. However, some of the results of section III.4 about 'second term identities' have not appeared elsewhere. In the last section I have explained how some of our results may be translated into a more classical language.

This article is an expansion of the two hour lecture I gave at RIMS, Kyoto in January of 1992 as part of the conference Studies on Automorphic Forms and Associated $L$-Functions, organized by K. Takase. I would like to thank Takase for his fine job in organizing the conference and for his essential assistance in arranging my visit to Japan. I would like to thank T. Oda, T. Yamazaki and N. Kurokawa for their hospitality in Kyoto, Fukuoka and Tokyo respectively. Finally I would like to express my gratitude to Japan Association for Mathematical Sciences (JAMS) for the generous Grant which made my very profitable visit possible.

\section{I.1. Background.}

We will work adelically and recall some of the basic machinery of theta functions, Eisenstein series, etc.

Let $F$ be a totally real number field and fix a non-trivial additive character $\psi$ of $F_{\mathrm{A}} / F$. Let $V,($,$) be a non-degenerate inner product space over F$ with $\operatorname{dim}_{F} V=m$. 
For convenience, we will assume that $m$ is even. Associated to $V$ is a quadratic character $\chi_{V}$ of $F_{\mathrm{A}}^{\times} / F^{\times}$,

$$
\chi_{V}(x)=\left(x,(-1)^{\frac{m}{2}} \operatorname{det}(V)\right)_{F}
$$

where $(,)_{F}$ is the Hilbert symbol of $F$ and $\operatorname{det}(V) \in F^{\times} / F^{\times, 2}$ is the determinant of the matrix $\left(\left(x_{i}, x_{j}\right)\right)$ for any basis $x_{1}, \ldots, x_{m}$ of $V$. Let $H=O(V)$ be the orthogonal group of $V$. Also let $W=F^{2 n}$ (row vectors), $<,>$ be the standard symplectic vector space over $F$ and let $G=S p(W)=S p(n)$ be the corresponding symplectic group. The Siegel parabolic $P=M N$ of $G$ is the subgroup which stabilizes the maximal isotropic subspace $\left\{(0, y) \mid y \in F^{n}\right\}$. It has a Levi factor

$$
M=\left\{m(a)=\left(\begin{array}{cc}
a & 0 \\
0 & { }^{t} a^{-1}
\end{array}\right) \mid a \in G L(n)\right\},
$$

and unipotent radical

$$
N=\left\{n(b)=\left(\begin{array}{ll}
1 & b \\
0 & 1
\end{array}\right) \mid b={ }^{t} b \in M(n)\right\} .
$$

For each non-archimedean place $v$ of $F$, let $K_{v}=S p\left(n, \mathcal{O}_{v}\right)$ where $\mathcal{O}_{v}$ is the ring of integral elements in the completion $F_{v}$. If $v$ is an archimedean place of $F$ let

$$
K_{v}=\left\{\left(\begin{array}{cc}
a & b \\
-b & a
\end{array}\right) \mid a+i b \in U(n)\right\} .
$$

Then let $K=\prod_{v} K_{v}$ be the corresponding maximal compact subgroup of $G(\mathrm{~A})$.

Let $\mathbb{W}=V \otimes_{F} W \simeq V^{2 n}$ with symplectic form $<<,>>=(,) \otimes<,>$, so that $(G, H)$ is a dual reductive pair in $S p(\mathbb{W})$.

Let $M p(\mathbb{W}) \longrightarrow S p(\mathbb{W})(\mathbb{A})$ be the metaplectic cover of $S p(\mathbb{W})(\mathbb{A})$ and let $\omega=\omega_{\psi}$ be the Weil representation [40] of $M p(\mathbb{W})$ associated to $\psi$. Since we are assuming that $m=\operatorname{dim}_{F}(V)$ is even, there is a splitting

$$
\begin{array}{ccc} 
& M p(\mathbb{W}) \\
& \nearrow & \downarrow \\
& & \\
& & \\
& & S p(\mathbb{N})(\mathrm{A})
\end{array}
$$

and $\omega$ yields a representation of $G(\mathrm{~A}) \times H(\mathrm{~A})$. This can be realized on the space $S\left(V(\mathbb{A})^{n}\right)$ of Schwartz-Bruhat functions on $V(\mathbb{A})^{n}$ with

$$
\begin{aligned}
(\omega(h) \varphi)(x) & =\varphi\left(h^{-1} x\right) & & h \in H(\mathbb{A}) \\
(\omega(m(a)) \varphi)(x) & =\chi_{V}(x)|\operatorname{det}(a)|_{\AA}^{\frac{m}{2}} \varphi(x a) & & a \in G L_{n}(\mathbb{A}) \\
(\omega(n(b)) \varphi)(x) & =\psi(\operatorname{tr}(b Q(x))) \varphi(x) & & b \in S y m_{n}(\mathbb{A}) .
\end{aligned}
$$


Here $Q(x)=\frac{1}{2}(x, x)$ is the value on $x \in V(\mathbb{A})^{n}$ of the quadratic form $Q$ associated to $($,$) .$

\section{I.2. Averages of theta functions.}

¿From this machinery we can construct the usual theta function. For $g \in G(\mathbb{A})$, $h \in H(\mathrm{~A})$ and $\varphi \in V(\mathrm{~A})^{n}$, let

$$
\theta(g, h ; \varphi)=\sum_{x \in V(F)^{n}}(\omega(g) \varphi)\left(h^{-1} x\right)
$$

This function is left invariant under $G(F)$ (Poisson summation) and $H(F)$ and is slowly increasing on $G(F) \backslash G(\mathrm{~A})$ and $H(F) \backslash H(\mathrm{~A})$. The average value with respect to $H$ is given by the integral

$$
I(g ; \dot{\varphi})=\int_{H(F) \backslash H(\mathbb{A})} \theta(g, h ; \varphi) d h
$$

where $d h$ is the invariant measure on $H(F) \backslash H(\mathbb{A})$ normalized to have total volume 1 (we exclude the case of a split binary space $V$ ). Weil's convergence criterion [41] asserts that $I(g ; \varphi)$ is absolutely convergent for all $\varphi$ provided

$$
\left\{\begin{array}{l}
(1) \quad V \text { is anisotropic or } \\
(2) \quad m-r>n+1
\end{array}\right.
$$

where $r$ is the Witt index of $V$, i.e., the dimension of a maximal isotropic $F$-subspace of $V$. Note that when $V$ is split, $m=2 r$ and condition (I.2.3)(2) becomes $m>2 n+2$. When $(\mathrm{I} .2 .3)(1)$ or $(\mathrm{I} .2 .3)(2)$ is satisfied, then $I(g ; \varphi)$ is an automorphic form on $G(\mathrm{~A})$, provided we assume that the function $\varphi$ is $K$-finite.

\section{I.3. Siegel's Eisenstein series.}

The Eisenstein series involved in the Siegel-Weil formula are defined as follows: The group $G(\mathrm{~A})$ has an Iwasawa decomposition

$$
G(\mathrm{~A})=P(\mathrm{~A}) K \text {. }
$$

Write any $g \in G(\mathrm{~A})$ as $g=n m(a) k$, and set

$$
|a(g)|=|\operatorname{det}(a)|_{\boldsymbol{A}} .
$$


This is a well defined function on $G(\mathrm{~A})$ which is left $N(\mathrm{~A}) M(F)$-invariant and right $K$-invariant. For $s \in \mathbb{C}$ and $\varphi \in S\left(V(\mathbb{A})^{n}\right)$, set

$$
\Phi(g, s)=\omega(g) \varphi(0) \cdot|a(g)|^{s-s_{0}}
$$

where

$$
s_{0}=\frac{m}{2}-\rho_{n}
$$

with $\rho_{n}=\frac{n+1}{2}$. Note that since

$$
\omega(n m(a) g) \varphi(0)=\chi_{V}(\operatorname{det}(a))|\operatorname{det}(a)|^{\frac{m}{2}} \omega(g) \varphi(0)
$$

we have

$$
\Phi(n m(a) g, s)=\chi_{V}(\operatorname{det}(a))|\operatorname{det}(a)|^{s+\rho_{n}} \Phi(g, s) .
$$

The space of all smooth $K$-finite functions $\Phi(s)$ on $G($ A $)$ with such a transformation on the left form an induced representation

$$
I_{n}\left(s, \chi_{V}\right)=\operatorname{Ind} d_{P(\mathbf{A})}^{G(\mathbf{A})}\left(\chi_{V} \cdot||^{s}\right), \quad \text { (normalized induction) }
$$

of $G(\mathrm{~A})$. It is important to note that the map

$$
\varphi \mapsto \Phi\left(s_{0}\right)
$$

defines a $G($ A $)$ intertwining map:

$$
S\left(V(\mathrm{~A})^{n}\right) \longrightarrow I_{n}\left(s_{0}, \chi_{V}\right)
$$

This is not true for other values of $s$ and explains why the point $s=s_{0}$ is the critical one in what follows. Moreover, this map is $H(\mathrm{~A})$-invariant, i.e., $\omega(h) \varphi$ and $\varphi$ have the same image. These two facts will play a key role.

Now let $\chi$ be any unitary character of $F_{\mathrm{A}}^{\times} / F^{\times}$and let $I_{n}(s, \chi)$ be the induced representation defined with $\chi$ in place of $\chi_{V}$. Note that $I_{n}(s, \chi)$ is a (restricted) tensor product $I_{n}(s, \chi)=\otimes_{v} I_{n, v}\left(s, \chi_{v}\right)$ of the corresponding local induced representations. Note that any $\Phi(s) \in I_{n}(s, \chi)$ is determined by its restriction to $K$. A section $\Phi(s) \in I_{n}(s, \chi)$ - see [3] for an explanation of this terminology - will be called standard if its restriction 
to $K$ is independent of $s$ and factorizable if it has the form $\Phi(s)=\otimes_{v} \Phi_{v}(s)$ with $\Phi_{v}(s) \in I_{n, v}\left(s, \chi_{v}\right)$.

For any $\Phi(s) \in I_{n}(s, \chi)$ and $g \in G(\mathrm{~A})$, the Siegel Eisenstein series is defined by:

$$
E(g, s, \Phi)=\sum_{\gamma \in P(F) \backslash G(F)} \Phi(\gamma g, s)
$$

which converges absolutely for $\operatorname{Re} s>\rho_{n}$, provided $\Phi(s)$ is standard, and defines an automorphic form on $G(\mathrm{~A})$. The general results of Langlands (cf. [3]) imply that $E(g, s, \Phi)$ has a meromorphic analytic continuation to the whole $s$-plane and satisfies a functional equation

$$
E(g, s, \Phi)=E(g,-s, M(s) \Phi)
$$

where

$$
M(s): I_{n}(s, \chi) \longrightarrow I_{n}\left(-s, \chi^{-1}\right)
$$

is the global intertwining operator defined, for $\operatorname{Re} s>\rho_{n}$, by

$$
M(s) \Phi(g)=\int_{N(\text { A })} \Phi(n g, s) d n
$$

\section{I.4. Convergent Siegel-Weil formulas.}

The classical Siegel-Weil formula gives a relation between the two automorphic forms $I(g ; \varphi)$ and $E(g, s, \Phi)$ ( $\Phi(s)$ coming from $\varphi$ ) associated to $\varphi$ in the convergent range. Specifically, assume that $s_{0}=\frac{m}{2}-\rho_{n}>\rho_{n}$ so that $E(g, s, \Phi)$ is absolutely convergent at $s=s_{0}$. Note that this condition is equivalent to $m>2 n+2$ so that (2) above hold and $I(g ; \varphi)$ converges absolutely as well.

Theorem [38],[41]. Suppose that $\Phi(s)$ is associated to $\varphi \in S\left(V(\mathbb{A})^{n}\right)$ and that $m>$ $2 n+2$. Then

$$
E\left(g, s_{0}, \Phi\right)=I(g ; \varphi)
$$

Of course, Weil proved such an identity in much greater generality in [41] for dual reductive pairs defined by algebras with involutions. 
Main Problem. Extend this result beyond the convergent range.

The first such extension is the following:

Theorem, $[\mathbf{1 8}, 19,35]$. Assume that Weil's convergence condition (1) or (2) holds so that $I(g ; \varphi)$ is well defined for all $\left.\varphi \in S(V(A))^{n}\right)$. Suppose that $\Phi(s)$ is associated to $\varphi \in$ $S\left(V(\mathrm{~A})^{n}\right)$. Then

(i) $E(g, s, \Phi)$ is holomorphic at $s=s_{0}$, and

(ii)

$$
E\left(g, s_{0}, \Phi\right)=\kappa \cdot I(g ; \varphi) \quad \kappa= \begin{cases}1 & \text { if } m>n+1 \\ 2 & \text { if } m \leq n+1\end{cases}
$$

Of course, a number of special cases were known earlier. For example, Siegel himself considered the case $n=1, m=4,[38, \mathrm{I}$, no.22, p.443 and III, no.58] etc.

REMARKS: (1) The Eisenstein series is essentially local in nature, i.e., it is built out of the local components

$$
\Phi_{v}(g, s)=\omega_{v}(g) \varphi_{v}(0)|a(g)|_{v}^{s-s_{0}}
$$

associated to the local components of a factorizable $\varphi=\otimes_{v} \varphi_{v}$. These components can be varied independently and carry no global information about $V$. On the other hand, $I(g ; \varphi)$ is essentially global in nature, since it involves the theta distribution:

$$
\varphi \mapsto \sum_{x \in V(F)^{n}} \varphi(x)
$$

and thus the position of lattice points, etc. The identities above thus contain a link between local and global information. A number theorist might be reminded of the HasseWeil or motivic $L$-functions (which are built out of purely local data) and the elaborate conjectures about their special values (which involve global invariants). It may not be unreasonable to hope that the Siegel-Weil formula may contain information about such special values in certain cases.

(2) Obviously, the Siegel-Weil formula can be viewed either as (i) identifying the theta integral $I(g ; \varphi)$ as an Eisenstein series, or (ii) identifying the special value at $s=s_{0}$ of $E(g, s, \Phi)$ as a theta function, provided the defining data $\Phi(s)$ is associated to $\varphi$. For many applications (ii) is a better point of view. It suggests that we should consider the whole family of Eisenstein series $E(g, s, \Phi)$ for arbitrary data $\Phi(s) \in I_{n}(s, \chi)$. This point of view is particularly important outside of the convergent range and is more convenient from a representation theoretic standpoint. 


\section{II.1. Some local representation theory.}

We now consider some of the representation theory which lies behind the Siegel-Weil formula.

We first fix a place non-archimedean $v$ of $F$ and temporarily shift notation, writing $F$ for the completion of the old $F$ at $v$, and similarly writing $G=S p_{n}(F), H=O(V)$, etc.

We have a local Weil representation $\omega$ of $G \times H$ on the space $S\left(V^{n}\right)$ of SchwartzBruhat functions on $V^{n}$. The local analogue of the map (I.3.8) is given by

$$
\begin{aligned}
S\left(V^{n}\right) & \longrightarrow I_{n}\left(s_{0}, \chi_{V}\right) \\
\varphi & \mapsto \Phi\left(s_{0}\right),
\end{aligned}
$$

where

$$
\Phi(g, s)=\omega(g) \varphi(0) \cdot|a(g)|^{s-s_{0}} .
$$

The map (II.1.1) is $G$-intertwining and $H$-invariant. In fact:

THEorem. (Rallis [33]) Let

$$
R_{n}(V)=S\left(V^{n}\right)_{H}
$$

be the maximal quotient of $S\left(V^{n}\right)$ on which $H$ acts trivially. Then the map (II.1.1) induces an injection of $R_{n}(V)$ into $I_{n}\left(s_{0}, \chi_{V}\right)$ :

$$
\begin{gathered}
S\left(V^{n}\right) \\
\downarrow \\
R_{n}(V):=S\left(V^{n}\right)_{H} \stackrel{\searrow}{\hookrightarrow} I_{n}\left(s_{0}, \chi_{V}\right) .
\end{gathered}
$$

Thus $R_{n}(V)$ may be identified with a submodule of $I_{n}\left(s_{0}, \chi_{V}\right)$.

Recall that the non-degenerate quadratic spaces over $F$ are classified by the invariants: $\operatorname{dim}_{F} V=m$, det $V \in F^{\times} / F^{\times, 2}$ and $\epsilon(V)$, the Hasse invariant of $V$. Note that det $V$ and the quadratic character $\chi_{V}$ determine each other uniquely. Thus, if we view $s_{0}=$ $\frac{m}{2}-\rho_{n}$ and $\chi=\chi_{V}$ as fixed, and if $m>2$, there are precisely two corresponding quadratic spaces $V_{ \pm}$, having opposite Hasse invariants. When $m=2$ there are again two spaces $V_{ \pm}$if $\chi \neq 1$, while, if $\chi=1$ there is only the split binary space $V_{\epsilon}$, where $\epsilon=(-1,-1)_{F}$, where $(,)_{F}$ is the quadratic Hilbert symbol for $F$. The induced 
representation $I_{n}\left(s_{0}, \chi\right)$ thus has two (resp. one, if $m=2$ and $\chi=1$ ) $G$-submodules, $R_{n}\left(V_{ \pm}\right)$. It turns out that these submodules account for all of the reducibility of $I_{n}\left(s_{0}, \chi\right)$. To state the precise result it is convenient to fix a generator $\varpi$ for the maximal ideal of the ring of integers $\mathcal{O}$ of $F$ and to call a character $\chi$ of $F^{\times}$normalized if $\chi(\varpi)=1$.

Theorem. ([11], [21]) Assume that $\chi$ is a normalised character of $F^{\times}$. Then

(1) If $\chi^{2} \neq 1$, then $I_{n}(s, \chi)$ is irreducible for all $s$.

(2) If $\chi^{2}=1$ and $\chi \neq 1$, then $I_{n}(s, \chi)$ is irreducible except at the points $s$ in the set

$$
\frac{m}{2}-\rho_{n}+\frac{i \pi}{\log q} \mathbb{Z} \quad 2 \leq m \leq 2 n, m \text { even. }
$$

(3) If $\chi=1$, then $I_{n}(s, \chi)$ is irreducible except at the points $s$ in the set

$$
\frac{m}{2}-\rho_{n}+\frac{i \pi}{\log q} \mathbb{Z} \quad 2 \leq m \leq 2 n, m \text { even }
$$

and at the points $s= \pm \rho_{n}+\frac{2 i \pi}{\log q} \mathbb{Z}$ corresponding to $m=0$ or $2 n+2$.

Here $q$ is the order of the residue class field of $F$.

Such induced representations were also considered in [14].

Note that, in cases (2) and (3), the vertical translation by $\frac{i \pi}{\log q}$ should be thought of as a shift of $\chi$ to the quadratic character $\chi \cdot \|^{\frac{i \pi}{\log q}}$. Thus, in the rest of our discussion, we will consider only quadratic characters $\chi$, without the assumption that $\chi$ is normalized, and will describe the submodule structure of $I_{n}(s, \chi)$ at a point $s_{0}=\frac{m}{2}-\rho_{n}$ with $0 \leq m \leq 2 n+2, m$ even.

The simplest case occurs when $n$ is odd and $m=n+1$, since the representation $I_{n}(0, \chi)$ is unitarizable and hence completely reducible. In this case, if $n>1$, or if $n=1$ and $\chi \neq 1$,

$$
I_{n}(0, \chi)=R_{n}\left(V_{+}\right) \oplus R_{n}\left(V_{-}\right)
$$

and the representations $R_{n}\left(V_{ \pm}\right)$are irreducible. In the case $n=1$ and $\chi=1$,

$$
I_{1}(0,1)=R_{n}\left(V_{\epsilon}\right)
$$

is irreducible.

If $2 \leq m<n+1$, the representations $R_{n}(V)$ are irreducible and distinct. In this range, excluding the case $m=2, \chi=1$, the subspace $R_{n}\left(V_{+}\right) \oplus R_{n}\left(V_{-}\right)$is the maximal 
proper submodule of $I_{n}\left(s_{0}, \chi\right)$. If $m=2$ and $\chi=1$, then $R_{n}(V)$ for the split binary space $V$ is the unique submodule of $I_{n}\left(s_{0}, 1\right)$. Similarly, if $m=0$ and $\chi=1$, so that $s_{0}=-\rho_{n}$, the trivial representation $\mathbb{1}=R_{n}(0)$ is the unique submodule of $I_{n}\left(-\rho_{n}, 1\right)$.

On the right hand side of the unitary axis, i.e., in the range $n+1<m \leq 2 n+2$, the situation is reversed. First of all, excluding the cases $\chi=1$ and $m=2 n$ or $2 n+2$, each of the spaces $V_{ \pm}$can be written as an orthogonal direct sum

$$
V_{ \pm}=V_{0, \pm}+V_{r, r}
$$

where $V_{0, \pm}$ is a quadratic space with the same Hasse invariant and determinant as $V_{ \pm}$ and of dimension $m_{0}=\operatorname{dim}_{F} V_{0, \pm}$ with

$$
m+m_{0}=2 n+2 \text {. }
$$

Here $V_{r, r}$ is a split space of dimension $2 r$, i.e., an orthogonal direct sum of $r$ hyperbolic planes.

Definition. The spaces $V_{ \pm}$and $V_{0, \pm}$ related in this way will be called complementary

Note that $R_{n}\left(V_{0, \pm}\right) \subset I_{n}\left(-s_{0}, \chi\right)$. Then $R_{n}\left(V_{ \pm}\right)$has a unique irreducible quotient $R_{n}\left(V_{0, \pm}\right)$, and the kernel of the map

$$
R_{n}\left(V_{ \pm}\right) \longrightarrow R_{n}\left(V_{0, \pm}\right)
$$

is irreducible [21]. The two submodules $R_{n}\left(V_{+}\right)$and $R_{n}\left(V_{-}\right)$span $I_{n}\left(s_{0}, \chi\right)$ and intersect in this irreducible submodule.

In the excluded cases $\chi=1$ and $m=2 n$ or $2 n+2$, the split space has Hasse invariant $\epsilon:=(-1,-1)^{\frac{n(n+1)}{2}}$ if $m=2 n$ or $(-1,-1)^{\frac{(n+1)(n+2)}{2}}$ if $m=2 n+2$. Again we have

$$
V_{\epsilon}=V_{0, \epsilon}+V_{r, r}
$$

and $R_{n}\left(V_{\epsilon}\right)$ has a unique irreducible quotient $R_{n}\left(V_{0, \epsilon}\right)$. Now, however, $R_{n}\left(V_{-\epsilon}\right)$ is the irreducible submodule of $R_{n}\left(V_{\epsilon}\right)$ which is the kernel of the map

$$
R_{n}\left(V_{\epsilon}\right) \longrightarrow R_{n}\left(V_{0, \epsilon}\right) .
$$


It is important to note that the representations $I_{n}\left(s_{0}, \chi\right)$ and $I_{n}\left(-s_{0}, \chi\right)$ are contragradient [33] and are, moreover, related by the normalized local intertwining operator

$$
M^{*}\left(s_{0}\right): I_{n}\left(s_{0}, \chi\right) \longrightarrow I_{n}\left(-s_{0}, \chi\right)
$$

This operator is a 'normalized' version of the global intertwining operator defined by (I.3.12),

$$
M^{*}(s)=\frac{1}{a_{n}(s, \chi)} M(s)
$$

where

$$
a_{n}(s, \chi)=L\left(s+\rho_{n}-n, \chi\right) \cdot \prod_{k=1}^{\left[\frac{n}{2}\right]} L\left(2 s-n+2 k, \chi^{2}\right) .
$$

Here $L(s, \chi)=\left(1-\chi(\varpi) q^{-s}\right)^{-1}$ is the local Euler factor when $\chi$ is unramified and is 1 if $\chi$ is ramified. In particular, the maps (II.1.5) and (II.1.7) can be realized as the restrictions of $M^{*}\left(s_{0}\right)$ to the subspace $R_{n}\left(V_{ \pm}\right)[21]$.

Two additional facts will be relevant [21].

(1) If $V$ is a split space, then the representation $R_{n}(V) \subset I_{n}\left(s_{0}, \chi_{V}\right)$ contains the normalized 'spherical' vector $\Phi^{0}\left(s_{0}\right)$ defined by requiring that

$$
\Phi^{0}\left(k, s_{0}\right)=1 .
$$

(2) If $m>2 n+2$, then $R_{n}\left(V_{ \pm}\right)=I_{n}\left(s_{0}, \chi_{V}\right)$.

Next we turn to the case of a real archimedean place $v$, which we again omit from the notation. We let $\mathfrak{g}($ resp. $\mathfrak{h})$ denote the Lie algebra of $G=S p(n, \mathbb{R})$ (resp. $H=O(V)$ ). Suppose that $\operatorname{sig} V=(p, q)$ with $p+q=m$, and fix an orthogonal decomposition

$$
V=U_{+}+U_{-}
$$

where $\operatorname{dim} U_{+}=p, \operatorname{dim} U_{-}=q$ and $\left.()\right|_{,U_{+}}\left(\left.\operatorname{resp.}()\right|_{,U_{-}}\right)$is positive (resp. negative) definite. Then $H \simeq O(p, q)$, and we let $K_{H} \simeq O(p) \times O(q)$ be the maximal compact subgroup of $H$ which preserves the decomposition (II.1.10). Let $\mathcal{S}\left(V^{n}\right)$ be the Schwartz space of $V^{n}$ and define the Gaussian $\varphi^{0} \in \mathcal{S}\left(V^{n}\right)$ by $\varphi^{0}(x)=\exp \left(-\pi(x, x)_{+}\right)$, where $\left.(,)_{+}\right|_{U_{+}}=($,$) and \left.(,)_{+}\right|_{U_{-}}=-($,$) . Then let S\left(V^{n}\right) \subset \mathcal{S}\left(V^{n}\right)$ be the space of all functions of the form $\varphi(x)=p(x) \varphi^{0}(x)$ where $p$ is a polynomial on $V^{n}$. The Weil representation of $G \times H$ can be realized on $\mathcal{S}\left(V^{n}\right)$ and $S\left(V^{n}\right)$ then becomes a $(\mathfrak{g}, K) \times\left(\mathfrak{h}, K_{H}\right)$ module (Harish-Chandra module). Note that $S\left(V^{n}\right)$ consists of $K \times K_{H^{-}}$ finite functions. Similarly, we let $I_{n}(s, \chi)$ denote the space of smooth $K$-finite functions on $G$ which satisfy (I.3.5). 
Theorem. ([20]) Let $R_{n}(p, q)$ be the maximal quotient of $S\left(V^{n}\right)$ on which $\left(\mathfrak{h}, K_{H}\right)$ acts trivially. Then the map (II.1.1) induces an injection of $R_{n}(p, q)$ into $I_{n}\left(s_{0}, \chi_{V}\right)$ :

$$
\begin{gathered}
S\left(V^{n}\right) \\
\downarrow \\
R_{n}(p, q):=S\left(V^{n}\right)_{H} \hookrightarrow I_{n}\left(s_{0}, \chi_{V}\right) .
\end{gathered}
$$

Thus $R_{n}(p, q)$ may be identified with a submodule of $I_{n}\left(s_{0}, \chi_{V}\right)$.

Note that then

$$
\chi_{V}(x)=\left(x,(-1)^{\frac{m}{2}+q}\right)_{\mathbb{R}} .
$$

Thus, if a quadratic character $\chi\left(\chi(x)=(\operatorname{sgn} x)^{a}\right.$ with $a=0$ or 1$)$ and a point $s_{0}=\frac{m}{2}-\rho_{n}$ are fixed, then each pair $(p, q)$ with $p+q=m$ and with $\chi(-1)=(-1)^{\frac{p-q}{2}}$ determines a submodule $R_{n}(p, q)$ of $I_{n}\left(s_{0}, \chi\right)$.

The reducibility and submodule structure of $I_{n}(s, \chi)$ is now considerably more complicated than in the $p$-adic case. The reducibility, for example, is competely determined in [20]. Here we simply record certain useful facts.

(1) The $(\mathfrak{g}, K)$-module $R_{n}(p, q)$ is generated by the vector $\Phi^{\ell}\left(s_{0}\right)$ whose value on $K \simeq U(n)$ is given by

$$
\Phi^{\ell}(k, s)=(\operatorname{det} k)^{\ell}
$$

(2) $R_{n}(p, q)$ is irreducible if $p+q=m \leq n+1$.

(3) On the unitary axis we have

$$
I_{n}(0, \chi)=\bigoplus_{\substack{p+q=m \\ \chi(-1)=(-1)^{\frac{p-q}{2}}}} R_{n}(p, q)
$$

(4) $R_{n}(m, 0)$ (resp. $R_{n}(0, m)$ ) is an irreducible lowest (resp. highest) weight representation of $(\mathfrak{g}, K)$.

We omit the case of a complex archimedean place. At the moment, we do not know the structure of $I_{n}(s, \chi)$, although it is undoubtedly known to the experts.

REMARK: The representation $R_{n}(V)$ always has a unique irreducible quotient, as it must by the local Howe duality principle [13], [28], [39]. This quotient is the representation $\theta(11)$ associated to the trivial representation of $H=O(V)$ via the local theta correspondence. 


\section{II.2. Some automorphic representations.}

We return to the global situation and assemble the local pieces. We assume that $\chi$ is a quadratic character of $F_{\mathrm{A}}^{\times} / F^{\times}$. Note that the global induced representation has a factorization:

$$
I_{n}(s, \chi)=\otimes_{v} I_{n, v}\left(s, \chi_{v}\right)
$$

as a representation of $\left(\mathfrak{g}, K_{\infty}\right) \times G\left(\mathbb{A}_{f}\right)$. Here we have taken $I_{n}(s, \chi)$ to consist of smooth, $K$-finite functions on $G(A)$, and the tensor product on the right side is the restricted tensor product with respect to the vectors $\Phi_{v}^{0}(s)$ defined by (II.1.10). For certain values of $s$ and $\chi$ the representation $I_{n}(s, \chi)$ has infinitely many constituents. For example, if $V$ is a quadratic space over $F$, as in section I above, we let $R_{n, v}(V)=R_{n}\left(V_{v}\right)$ be the representation of $G_{v}$ if $v$ is non-archimedean (resp. $\left(\mathfrak{g}_{v}, K_{v}\right)$, if $v$ is archimedean) defined in section II.1. Since $V_{v}$ is split at almost all places, we may define the restricted tensor product

$$
\Pi_{n}(V)=\otimes_{v} R_{n, v}(V)
$$

and this representation is a submodule of $I_{n}\left(s_{0}, \chi_{V}\right)$.

\section{Examples:}

(1) If $m \leq n+1$, then $\Pi_{n}(V)$ is an irreducible submodule of $I_{n}\left(s_{0}, \chi_{V}\right)$. Note that $s_{0} \leq 0$.

(2) If $m>2 n+2$, then

$$
I_{n, f}(s, \chi)=\otimes_{v \text { finite }} I_{n, v}\left(s, \chi_{v}\right)
$$

is irreducible for all $s$, while $I_{n, \infty}(s, \chi)$ is irreducible unless $s=s_{0}=\frac{m}{2}-\rho_{n}$ for some $m \in 2 \mathbb{Z}$. At such points $s_{0}, I_{n, \infty}\left(s_{0}, \chi\right)$ and hence $I_{n}\left(s_{0}, \chi\right)$ has finite length.

(3) In the range $n+1<m \leq 2 n+2, I_{n}\left(s_{0}, \chi\right)$ has infinite length and has no irreducible admissible submodules.

In fact, not every submodule of $I_{n}\left(s_{0}, \chi\right)$ is associated to a quadratic space. The key examples are defined as follows. 
Definition. Fix a quadratic character $\chi$ and an even integer $m \geq 0$. A collection $\mathcal{C}=\left\{U_{v}\right\}$ of quadratic spaces $U_{v}$ over $F_{v}$, one for each place $v$, will be called an incoherent family if

(i) $\operatorname{dim}_{F_{v}} U_{v}=m$ and $\chi_{U_{v}}=\chi$.

(ii) For almost all $v U_{v}$ is the split space of dimension $m$.

(iii)

$$
\prod_{v} \epsilon_{v}\left(U_{v}\right)=-1
$$

where $\epsilon_{v}\left(U_{v}\right)$ is the Hasse invariant of $U_{v}$.

In particular, there cannot be a global quadratic space $V$ over $F$ whose localizations are isomorphic to the $U_{v}$ 's since condition (iii) violates the product formula for the Hasse invariants of such localizations.

Note that, if we exclude the case $m=2$, then an incoherent family $\mathcal{C}=\left\{U_{v}\right\}$ when modified at any one place $v$ (by switching $U_{v}$ to the space $U_{v}^{\prime}$ with the opposite Hasse invariant) becomes the set of localizations $\left\{V_{v}\right\}$ of a global quadratic space $V$ of dimension $m$ and character $\chi_{V}=\chi$. No particular place $v$ is 'prefered'. In the case $m=2$ with $\chi \neq 1$ we can only modify at the places $v$ at which $\chi_{v} \neq 1$. When $m=2$ and $\chi=1$ there are no incoherent families.

If $\mathcal{C}$ is an incoherent family, we can form, thanks to (ii), the representation

$$
\Pi_{n}(\mathcal{C})=\otimes_{v} R_{n}\left(U_{v}\right)
$$

and, when $m \leq n+1$, this representation is an irreducible submodule of $I_{n}\left(s_{0}, \chi\right)$. In fact, in the range $-\rho_{n} \leq s_{0} \leq n+1$, every irreducible submodule is either a $\Pi_{n}(V)$ or a $\Pi_{n}(\mathcal{C})$. Note that we could formally include the case $m=0$ and obtain $\Pi_{n}(0)=\mathbb{1}$, the trivial representation.

Now since $\chi$ is automorphic, i.e., is a character of $F_{\mathrm{A}}^{\times} / F^{\times}$and not just of $F_{\mathrm{A}}^{\times}$, it follows from a very general result of Langlands [25] that every irreducible constituent of $I_{n}(s, \chi)$ occurs as a subquotient of the space of automorphic forms on $G(A)$. This result is proved using the general theory of Eisenstein series and their derivatives. For the representations $\Pi_{n}(V)$ and $\Pi_{n}(\mathcal{C})$ we can give much more explicit information.

For example, fix a global quadratic space $V$ and assume that Weil's convergence condition (I.2.3) holds. Then the theta integral (I.2.2) defines an intertwining map for the 
$(\mathfrak{g}, K) \times G\left(\mathbb{A}_{f}\right)$ action,

$$
\begin{array}{cc}
S\left(V(\mathbb{A})^{n}\right) & \\
\downarrow & \searrow \\
\bar{I}: \quad \Pi_{n}(V) & \longrightarrow \mathcal{A}(G),
\end{array}
$$

where $\mathcal{A}(G)$ is the space of automorphic forms on $G(\mathrm{~A})$.

On the other hand, for $s_{0}>\rho_{n}$, i.e., for $m>2 n+2$, the Siegel Eisenstein series is termwise absolutely convergent for all $\Phi(s) \in I_{n}(s, \chi)$, and hence defines an intertwining map

$$
\Pi_{n}(V) \subset I_{n}\left(s_{0}, \chi\right) \stackrel{E\left(s_{0}\right)}{\longrightarrow} \mathcal{A}(G)
$$

REMARK: The injectivity of $E\left(s_{0}\right)$ can be proved by considering the constant term of $E\left(g, s_{0}, \Phi\right)$ as in [18]. The 'exponents' of the $n+1$ terms in this constant term are distinct in the range $\operatorname{Re}(s)>\rho_{n}$ so that there can be no cancellations among them. On the other hand, the first term is just $\Phi(g, s)$ itself.

The classical Siegel-Weil formula says that the two maps $\bar{I}$ and $E\left(s_{0}\right)$ agree on $\Pi_{n}(V)$ ! Thus, from a representation theoretic viewpoint, the classical Siegel-Weil formula expresses an Eisenstein intertwining map in terms of a theta intertwining map. This interpretation has a nice extension to the divergent range.

REMARK: Note that it follows that $\bar{I}$ is injective on $\Pi_{n}(V)$.

We next suppose that $m \leq n$, so that $\Pi_{n}(V)$ and $\Pi_{n}(\mathcal{C})$ are irreducible.

TheOREM, [23].

(i) In the range $0 \leq m \leq n$,

$$
\operatorname{dim} \operatorname{Hom}_{G(\mathbb{A})}\left(\Pi_{n}(V), \mathcal{A}(G)\right)=1
$$

Moreover, excluding the case $m=2$ and $\chi=1$, the representations $\Pi_{n}(V)$ are square integrable, i.e., occur as invariant subspaces in $\mathcal{A}_{(2)}(G)$, the space of square integrable automorphic forms.

(ii) If $2 \leq m \leq n+1$, and $\mathcal{C}$ is an incoherent family, then

$$
H o m_{G(\mathbb{A})}\left(\Pi_{n}(\mathcal{C}), \mathcal{A}(G)\right)=0
$$


i.e., the irreducible representation $\Pi_{n}(\mathcal{C})$ does not occur as an invariant subspace of $\mathcal{A}(G)$. It only occurs as a subquotient.

This uniqueness result is the key to Siegel-Weil formula in the divergent range.

\section{III.1. Poles of the Siegel Eisenstein series.}

The Siegel Eisenstein series defined using a section $\Phi(s)$ associated to a function $\varphi \in$ $S\left(V(\mathrm{~A})^{n}\right)$ where $V$ satisfies Weil's convergence condition (I.2.3) is always holomorphic at the point $s_{0}=\frac{m}{2}-\rho_{n}$. On the other hand, if $\Phi(s)$ is an arbitrary section and if $0 \leq m \leq 2 n+2, E(g, s, \Phi)$ can indeed have a pole. We can give a rather complete description of these poles.

The first step is to normalize the Eisenstein series. Let $\Phi(s)=\otimes_{v} \Phi_{v}(s) \in I_{n}(s, \chi)$ be a factorizable standard section (cf. I.3), and let $S$ be a finite set of places, including all of the archimedean places, such that, for any $v \notin S, \Phi_{v}(s)$ is the normalized spherical vector of (II.1.10). For any place $v$ of $F$, let

$$
b_{n, v}(s, \chi)=L_{v}\left(s+\rho_{n}, \chi_{v}\right) \cdot \prod_{k=1}^{\left[\frac{n}{2}\right]} L_{v}\left(2 s+n+1-2 k, \chi^{2}\right)
$$

where $L_{v}(s, \chi)$ is the local $L$-factor of $\chi$, and let

$$
b_{n}^{S}(s, \chi)=\prod_{v \notin S} b_{n, v}(s, \chi)
$$

The normalized Eisenstein series is then

$$
E^{*}(g, s, \Phi)=b_{n}^{S}(s, \chi) E(g, s, \Phi)
$$

Theorem[22,23]. Let $\Phi(s)$ be a standard section of $I_{n}(s, \chi)$, and let $S$ be as above.

(i) If $\chi^{2} \neq 1$, then $E^{*}(g, s, \Phi)$ is entire.

(ii) If $\chi^{2}=1$, then $E^{*}(g, s, \Phi)$ has at most simple poles, and these can only occur at the points $s_{0} \in X_{n}^{+}=X_{n} \cap \mathbb{P}_{>0}$, where

$$
X_{n}=\left\{-\rho_{n}, 1-\rho_{n}, \ldots \rho_{n}-1, \rho_{n}\right\}
$$

Moreover, if $\chi \neq 1$, then no pole occurs at $\rho_{n}$. 
Note that the normalizing factor $b^{S}(s, \chi)$ has no poles or zeroes in the right half plane, so that the behavior of $E(g, s, \Phi)$ in this half plane is the same as that of $E^{*}(g, s, \Phi)$.

To determine the residues, we fix a quadratic character $\chi$ and a point $s_{0}=\frac{m}{2}-\rho_{n} \epsilon$ $X_{n}^{+}$. The map

$$
\begin{aligned}
A_{-1}: I_{n}\left(s_{0}, \chi\right) & \longrightarrow \mathcal{A}(G) \\
\Phi\left(s_{0}\right) & \mapsto \operatorname{Res}_{s=s_{0}} E(g, s, \Phi),
\end{aligned}
$$

where $\Phi(s) \in I_{n}(s, \chi)$ is the standard extension of $\Phi\left(s_{0}\right) \in I_{n}\left(s_{0}, \chi\right)$, defines a $\left(\mathfrak{g}, K_{\infty}\right) \times$ $G\left(\mathrm{~A}_{f}\right)$ intertwining operator. Since for each place $v$ the local induced representation $I_{n, v}\left(s_{0}, \chi\right)$ is spanned by the $R_{n}\left(U_{v}\right)$ 's for $\operatorname{dim}_{F_{v}} U_{v}=m$ and $\chi_{U_{v}}=\chi_{v}$, we may as well assume that our section $\Phi(s)=\otimes_{v} \Phi_{v}(s)$ with $\Phi_{v}\left(s_{0}\right) \in R_{n}\left(U_{v}\right)$ for each $v$. Such a section $\Phi\left(s_{0}\right) \in \Pi_{n}\left(\left\{U_{v}\right\}\right)$ is called homogeneous; the collection of $U_{v}$ 's need not be unique. There are then two cases.

(1) The section $\Phi\left(s_{0}\right)$ is 'coherent', i.e., $\Phi\left(s_{0}\right) \in \Pi_{n}(V)$ for some global quadratic space $V$ over $F$.

(2) The section $\Phi\left(s_{0}\right)$ is incoherent, i.e., the only possible collections $\mathcal{C}=\left\{U_{v}\right\}$ such that $\Phi\left(s_{0}\right) \in \Pi_{n}(\mathcal{C})$ are incoherent families.

The resulting Eisenstein series will be called 'coherent' and 'incoherent' at the point $s_{0}$ respectively.

First consider the case of coherent sections associated to a quadratic space $V$. If Weil's condition (I.2.3) is satisfied, then $E(g, s, \Phi)$ is holomorphic at $s_{0}$ so the subspace $\Pi_{n}(V)$ lies in the kernel of the map $A_{-1}$. If, on the other hand, $V$ is isotropic with $m-r \leq n+1$ where $r$ is the Witt index of $V$, then (and only then!) there is an orthogonal decomposition

$$
V=V_{0}+V_{t, t}
$$

where

$$
\operatorname{dim}_{F} V+\operatorname{dim}_{F} V_{0}=2 n+2
$$

and $V_{t, t}$ is a split space of dimension $2 t$. Note that the localizations $V_{v}$ and $V_{0, v}$ will then be complementary in the sense defined in section II. In particular, there is a quotient map

$$
\Pi_{n}(V) \longrightarrow \Pi_{n}\left(V_{0}\right)
$$


given by the tensor product of the local quotient maps (II.1.5), (II.1.7), and their archimedean analogue.

REMARK: Since the existence of a complementary $V_{0}$ to $V$ is equivalent to the failure of Weil's convergence criterion (I.2.3) for $V$, we obtain a structural explanation of that criterion.

Theorem, [23]. Asume that $m=\operatorname{dim} V>n+1$.

(i) If $V$ satisfies the condition (I.2.3), then the restriction of $A_{-1}$ to $\Pi_{n}(V)$ is identically zero $[\mathbf{1 8}, 19]$.

(ii) If $V$ is isotropic with $m-r \leq n+1$, then the restriction of $A_{-1}$ to $\Pi_{n}(V)$ factors through the quotient map (III.1.7) and induces an injection

$$
\bar{A}_{-1}: \Pi_{n}\left(V_{0}\right) \hookrightarrow \mathcal{A}(G) .
$$

(iii) The restriction of $A_{-1}$ to $\Pi_{n}(\mathcal{C})$ for an incoherent family $\mathcal{C}$ is identically zero.

Finally, we consider the center of the critical strip when $n$ is odd. Now $E^{*}(g, s, \Phi)$ is holomorphic at $s=s_{0}=0$, for any standard section $\Phi(s)$. If we exclude the case $n=1$ and $\chi=1$, then the normalizing factor $b_{n}^{S}(s, \chi)$ is holomorphic and non-zero at $s=0$, so that we could just as well consider $E(g, s, \Phi)$ itself. Note that

$$
I_{n}(0, \chi)=\left(\bigoplus_{V} \Pi_{n}(V)\right) \oplus\left(\bigoplus_{\mathcal{C}} \Pi_{n}(\mathcal{C})\right)
$$

where $V$ runs over all (isomorphism classes of) quadratic spaces over $F$ of dimension $m=n+1$ and character $\chi_{V}=\chi$, and $\mathcal{C}$ runs over all incoherent families of the same type.

Theorem [23]. The map $A_{0}: I_{n}(0, \chi) \longrightarrow \mathcal{A}(G)$ given by

$$
A_{0}(g, \Phi)=E(g, 0, \Phi)
$$

induces an injection

$$
\overline{A_{0}}: \bigoplus \Pi_{n}(V) \hookrightarrow \mathcal{A}(G)
$$


Here $\operatorname{dim} V=m=n+1$. Moreover the restriction of $A_{0}$ to $\bigoplus \Pi_{n}(\mathcal{C})$ is zero.

Thus the residues (resp. values) of the Siegel Eisenstein series provide non-zero embeddings of the irreducible $\Pi_{n}(V)$ 's, $\operatorname{dim} V \leq n$ (resp. $\operatorname{dim} V=n+1$ when $n$ is odd) into $\mathcal{A}(G)$. This fact is not yet a Siegel-Weil formula, however. To obtain one we must next realize the $\Pi_{n}(V)$ 's as spaces of theta functions.

\section{III.2. Regularized theta integrals.}

Let $V$ be an isotropic quadratic space over $F$, with Witt index $r$, such that $m-r \leq$ $n+1$, so that the theta integral (I.2.2) may be divergent. We want to define a regularized version of this integral by using an old trick of Maass [27]: we apply a central differential operator to eliminate the 'bad terms' of the integrand. Similar operators were also found independently by Deitmar and Kreig [6].

Write

$$
V=V_{a n}+V_{r, r}
$$

where $V_{a n}$ is anisotropic, and assume that $\operatorname{dim}_{F} V=m \leq 2 n$. Recall that $W$ was the standard symplectic space over $F$ which defined $G=S p(W)=S p(n)$. Then there is another model of the Weil representation of $G(\mathbb{A}) \times H(\mathbb{A})$ on the space $S\left(V_{a n}(\mathbb{A})^{n}\right) \otimes$ $S\left(W(\mathrm{~A})^{r}\right)$, and the two models are related by a partial Fourier transform:

$$
S\left(V(\mathrm{~A})^{n}\right) \stackrel{\sim}{\longrightarrow} S\left(V_{a n}(\mathrm{~A})^{n}\right) \otimes S\left(W(\mathrm{~A})^{r}\right) \quad \varphi \mapsto \hat{\varphi} .
$$

Poisson summation implies that

$$
\sum_{x \in V(F)^{n}} \varphi(x)=\sum_{\substack{y \in V_{a n}(F)^{n} \\ w \in W(F)^{r}}} \hat{\varphi}(y, w) .
$$

Thus

$$
\theta(g, h ; \varphi)=\sum_{\substack{y \in V_{a n}(F)^{n} \\ w \in W(F)^{r}}} \hat{\omega}(g, h) \hat{\varphi}(y, w) .
$$

It turns out that the terms in this second expression for $\theta(g, h ; \varphi)$ which do not decay well on $H(F) \backslash H(\mathbb{A})$ are precisely those involving pairs $(y, w)$ with $w \in W(F)^{r} \simeq M_{r, 2 n}(F)$ having rank less than $r$. On the other hand, the sum of the terms involving $w$ 's of rank $r$ is rapidly decreasing on $H(F) \backslash H(\mathrm{~A})$. 
Theorem[23]. Fix an archimedean place $v$ of $F$. Then there exist elements $z \in \mathfrak{z}\left(\mathfrak{g}_{v}\right)$ and $z^{\prime} \in \mathfrak{z}\left(\mathfrak{h}_{v}\right)$, where $z\left(\mathfrak{g}_{v}\right)$ (resp. $\mathfrak{z}\left(\mathfrak{h}_{v}\right)$ ) is the center of the enveloping algebra of $\mathfrak{g}_{v}=\operatorname{Lie}_{v}\left(\right.$ resp. $\left.\mathfrak{h}_{v}=\operatorname{LieH}_{v}\right)$, such that

(i) $\omega(z)=\omega\left(z^{\prime}\right) \neq 0$.

(ii) For all $\varphi_{v} \in S\left(V_{a n, v}\right) \otimes S\left(W_{v}^{r}\right),(\omega(z) \varphi)(y, w)=0$ whenever the rank of $w \in W_{v}^{r} \simeq M_{r, 2 n}\left(F_{v}\right)$ is less than $r$.

Corollary. $\theta(g, h ; \omega(z) \varphi)$ is rapidly decreasing on $H(F) \backslash H(\mathrm{~A})$.

Then we can consider the integral

$$
\int_{H(F) \backslash H(\mathbb{A})} \theta(g, h ; \omega(z) \varphi) E\left(h, s^{\prime}\right) d h,
$$

where $E\left(h, s^{\prime}\right)$ is the Eisenstein series on $H(\mathrm{~A})$ associated to the parabolic subgroup which stabilizes a maximal isotropic subspace, and normalized so that $E\left(h, s^{\prime}\right)$ has constant residue 1 at the point $s^{\prime}=s_{0}^{\prime}=\rho^{\prime}$, analogous to $\rho_{n}$. This integral is absolutely convergent whenever $E\left(h, s^{\prime}\right)$ is holomorphic. For large $\operatorname{Re}\left(s^{\prime}\right)$ it can be unfolded in the usual way and turns out to be equal to

$$
P\left(s^{\prime} ; z\right) \cdot \mathcal{E}\left(g, s^{\prime}, \varphi\right)
$$

where $P\left(s^{\prime}, z\right)$ is an explicit polynomial in $s^{\prime}$ and $\mathcal{E}\left(g, s^{\prime}, \varphi\right)$ is an Eisenstein series on $G\left(\right.$ A) associated to a maximal parabolic subgroup $P_{r}$ of $G$ which stabilizes an isotropic $r$-plane in $W$. Then

$$
\mathcal{E}\left(g, s^{\prime}, \varphi\right)=\frac{1}{P\left(s^{\prime} ; z\right)} \int_{H(F) \backslash H(\mathbb{A})} \theta(g, h ; \omega(z) \varphi) E\left(h, s^{\prime}\right) d h .
$$

If $\varphi$ is such that $\theta(g, h ; \varphi)$ is already rapidly decreasing on $H(F) \backslash H(\mathbb{A})$, then

$$
\begin{aligned}
& \int_{H(F) \backslash H(\mathbb{A})} \theta(g, h ; \omega(z) \varphi) E\left(h, s^{\prime}\right) d h \\
= & \int_{H(F) \backslash H(\mathbb{A})} \omega\left(z^{\prime}\right) \cdot \theta(g, h ; \varphi) E\left(h, s^{\prime}\right) d h \\
= & \int_{H(F) \backslash H(\mathbb{A})} \theta(g, h ; \varphi) \omega\left(z^{\prime}\right)^{*} \cdot E\left(h, s^{\prime}\right) d h \\
= & P\left(s^{\prime} ; z\right) \cdot \int_{H(F) \backslash H(\mathbb{A})} \theta(g, h ; \varphi) E\left(h, s^{\prime}\right) d h
\end{aligned}
$$

so that

$$
\mathcal{E}\left(g, s^{\prime}, \varphi\right)=\int_{H(F) \backslash H(\mathbf{A})} \theta(g, h ; \varphi) E\left(h, s^{\prime}\right) d h
$$

in this case. 


\section{III.3. Extended Siegel-Weil formulas.}

We are interested in the Laurent expansion of $\mathcal{E}\left(g, s^{\prime}, \varphi\right)$ at the point $s_{0}^{\prime}$. The polynomial $P\left(s^{\prime} ; z\right)$ has the property that

$$
\underset{s_{0}^{\prime}}{\operatorname{ord}} P\left(s^{\prime} ; z\right)=\left\{\begin{aligned}
0 & \text { if } 2 \leq m \leq n+1 \\
+1 & \text { if } n+1<m \leq 2 n
\end{aligned}\right.
$$

Thus

$$
\underset{s_{0}^{\prime}}{\operatorname{ord}} \mathcal{E}\left(g, s^{\prime}, \varphi\right)= \begin{cases}+1 & \text { if } 2 \leq m \leq n+1 \\ +2 & \text { if } n+1<m \leq 2 n\end{cases}
$$

and so we have

$$
\mathcal{E}\left(g, s^{\prime}, \varphi\right)=\frac{B_{-2}(g, \varphi)}{\left(s^{\prime}-s_{0}^{\prime}\right)^{2}}+\frac{B_{-1}(g, \varphi)}{\left(s^{\prime}-s_{0}^{\prime}\right)}+O(1)
$$

We view the various terms in this expansion as the regularization of the theta integral (I.2.2).

Theorem[23]. Let $V$ be an isotropic quadratic space over $F$ with $m-r \leq n+1$.

(i) If $2 \leq m \leq n+1$, then the map $B_{-1}$ induces a diagram:

$$
S\left(V(\mathrm{~A})^{n}\right)
$$

(III.3.4)

$$
\begin{aligned}
& \downarrow \quad \searrow_{-1}^{B} \\
& \bar{I}=\bar{B}_{-1}: \quad \Pi_{n}(V) \quad \hookrightarrow \quad \mathcal{A}(G) \text {. }
\end{aligned}
$$

(ii) If $n+1<m \leq 2 n$, let $V_{0}$ be the complementary space to $V$. Then the map $B_{-2}$ factors through $\Pi_{n}(V)$ and further factors through the quotient $\Pi_{n}\left(V_{0}\right)$, i.e.,

$$
S\left(V(\mathrm{~A})^{n}\right)
$$

(III.3.5)

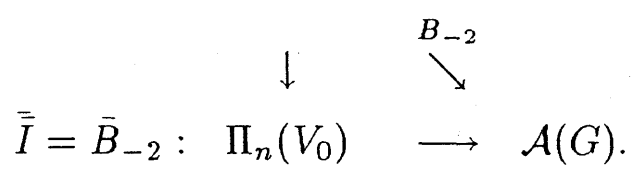

As a consequence of the uniqueness result of section II, we finally obtain a Siegel-Weil formula: 
Corollary [23]. (Siegel-Weil formula for residues and central values)

(i) Assume that $n+1<m \leq 2 n$ and let $V$ be an $m$ dimensional isotropic quadratic space over $F$ with $m-r \leq n+1$. Then there exists a non-zero constant $c_{1}$ such that

$$
\bar{A}_{-1}=c_{1} \cdot \bar{B}_{-1}=c_{1} \cdot \bar{I}
$$

where $\bar{A}_{-1}$ is the map on $\Pi_{n}\left(V_{0}\right)$ induced by the restriction of $A_{-1}$ to $\Pi_{n}(V)$.

(ii) Assume that $n$ is odd and that $n+1=m$ and let $V$ be an $m$ dimensional quadratic space over $F$. Then there exists a non-zero constant $c_{0}$ such that

$$
\left.A_{0}\right|_{\Pi_{n}(V)}= \begin{cases}c_{0} \cdot \bar{B}_{-1}=c_{0} \cdot \bar{I} & \text { if } V \text { is isotropic, } \\ 2 I & \text { if } V \text { is anisotropic. }\end{cases}
$$

Here the case $n=1$ and $\chi=1$ is excluded.

\section{III.4. Second term identities.}

In fact, one would like to obtain further relations among the terms of the Laurent expansions of the Siegel Eisenstein series $E(g, s, \Phi)$, for $\Phi(s)$ associated to $\varphi$, and those of the regularized theta integral $\mathcal{E}\left(g, s^{\prime}, \varphi\right)$. Specifically, we would like to consider

$$
E(g, s, \Phi)=\frac{A_{-1}(g, \Phi)}{s-s_{0}}+A_{0}(g, \Phi)+O\left(s-s_{0}\right)
$$

and to express $A_{0}$ and a combination of $B_{-1}$ and $B_{-2}$. So far this has only been done in some special cases.

Consider the case $n=2$ and $m=4$, so that $s_{0}=\frac{1}{2}$.

Theorem, [24]. There exists a constant $c^{\prime}$ such that

$$
A_{0}(g, \Phi)=c^{\prime} B_{-1}(g, \varphi)+B_{-2}\left(g, \varphi^{\prime}\right)
$$

where $\Phi(s)$ is the standard section associated to $\varphi$ and for some function $\varphi^{\prime} \in S\left(V(A)^{2}\right)$.

REMARK: Such a relation must be rather subtle since the map $\varphi \mapsto A_{0}(g, \Phi)$ is $H(A)$ invariant but it not (!!) $G(\mathrm{~A})$ intertwining (since the second term in the Laurent expansion is not $G(\mathrm{~A})$ intertwining (cf. $[\mathbf{1 9}, \S 2]$ ) while the map $\varphi \mapsto B_{-1}(g, \varphi)$ is $G(\mathrm{~A})$ intertwining but not (!!) $H(\mathbb{A})$ invariant. This explains the presence of the term $B_{-2}\left(g, \varphi^{\prime}\right)$ and the 
occurrence of the additional function $\varphi^{\prime}$. For example, if $\varphi$ is replaced by $\omega(h) \varphi$, the term $A_{0}(g, \Phi)$ is unchanged, but the term $B_{-1}(g, \varphi)$ only remains invariant modulo the subspace $\operatorname{Im}\left(B_{-2}\right) \subset \mathcal{A}(G)$. The convoluted arguments needed in section 6 of [24] reflect these difficulties.

REMARK: If $\Phi(s)$ is a standard section which is 'incoherent' at the point $s=\frac{1}{2}$, i.e, such that $\Phi\left(\frac{1}{2}\right) \in \Pi_{2}(\mathcal{C})$ for some incoherent family $\mathcal{C}$ but does not lie in any $\Pi_{n}(V)$, then the nature of $A_{0}(g, \Phi)$ remains to be determined.

A second term identity has also been proved is the case when $V=V_{r, r}$ is a split space and $\varphi \in S\left(V(\mathbb{A})^{n}\right)$ is invariant under $K$, the maximal compact subgroup of $G(\mathbb{A})$. In this case, the section $\Phi(s)$ associated to $\varphi$ is (up to a constant which we may assume to be 1 ) just

$$
\Phi(s)=\Phi^{0}(s)=\otimes_{v} \Phi_{v}^{0}(s)
$$

where $\Phi_{v}^{0}(s)$ is the normalized $K_{v}$-invariant standard section of (II.1.10) or (II.1.13) with $\ell=0$. On the other hand, the Eisenstein series $\mathcal{E}\left(g, s^{\prime}, \varphi\right)$ is also associated to a $K$ invariant (but not standard) section of an induced representation for the maximal parabolic $P_{r}$ of $G$.

More precisely, for any $r$ with $1 \leq r \leq n$ let $P_{r}$ be the maximal parabolic subgroup of $G=S p(n)$ which stabilizes the isotropic $r$-plane spanned by the vectors $e_{1}^{\prime}, \ldots, e_{r}^{\prime}$. Here we have fixed a standard symplectic basis $e_{1}, \ldots, e_{n}, e_{1}^{\prime}, \ldots, e_{n}^{\prime}$ for $W \simeq F^{2 n}$ (row vectors), so that $\left(\begin{array}{cc}0 & 1_{n} \\ -1_{n} & 0\end{array}\right)$ is the matrix for $\left.<,\right\rangle$. Then $P_{r}=N_{r} M_{r}$ where the Levi factor $M_{r} \simeq G L(r) \times S p(n-r)$. For the global Iwasawa decomposition $G(\mathrm{~A})=P_{r}(\mathrm{~A}) K$ we write $g=n_{r} m_{r}\left(a, g_{0}\right) k$ with $a \in G L(r, \mathbb{A})$ and $g_{0} \in S p(n-r, \mathbb{A})$ and we set

$$
\left|a_{r}(g)\right|=|\operatorname{det}(a)|_{\text {A }} .
$$

Let

$$
\mathcal{E}(g, s ; r, n)=\sum_{\gamma \in P_{r}(F) \backslash G(F)}\left|a_{r}(\gamma g)\right|^{s+\rho_{r, n}},
$$

where $\rho_{r, n}=n-\frac{r-1}{2}$. We normalize this series by setting

$$
\mathcal{E}^{*}(g, s ; r, n):=c_{r, n}(s) b_{r}(s) \mathcal{E}(g, s ; r, n)
$$


where $b_{r}(s)=b_{r}(s, \chi)$ for $\chi=1$ is defined by (III.1.1) with $S=\phi$ and

$$
c_{r, n}(s)=\prod_{i=0}^{r-1} L\left(s+\rho_{r, n}-i\right)
$$

Here we take the full Euler product, $L(s)=L(s, 1)=\prod_{v} L_{v}(s, 1)$, including the archimedean factors. Similarly, we let

$$
E^{*}\left(g, s, \Phi^{0}\right)=b_{n}(s, 1) E\left(g, s, \Phi^{0}\right)
$$

be the spherical Siegel Eisenstein series, normalised with the full Euler product $b_{n}(s, \chi)$ with $\chi=1$.

¿From now on we assume that $F=\mathbb{Q}$ so that $L(s)=L(1-s)$ and

$$
L(s)=\frac{1}{s-1}+\kappa+O(s-1)
$$

Note that the functional equation of $E^{*}\left(s, s, \Phi^{0}\right)$ is then simply

$$
E^{*}\left(g, s, \Phi^{0}\right)=E^{*}\left(g,-s, \Phi^{0}\right)
$$

because

$$
M(s) \Phi^{0}=\frac{a_{n}(s)}{b_{n}(s)} \Phi^{0}(-s)
$$

and $a_{n}(s)=b_{n}(-s)$. By a very elaborate inductive argument one can prove the following:

Theorem, [17]. (first term identities again) Let $s_{0}=r-\rho_{n}=r-\frac{n+1}{2}$.

(i) If $s_{0}<0$, then

$$
\operatorname{Res}_{s=s_{0}} E^{*}\left(g, s, \Phi^{0}\right)=c(r, n) \underset{s=\frac{r-1}{2}}{\operatorname{Res}} \mathcal{E}^{*}(g, s ; r, n) .
$$

(ii) If $s_{0}=0$, so that $n$ is odd, then

$$
E^{*}\left(g, 0, \Phi^{0}\right)=c(r, n) \operatorname{Res}_{s=\frac{r-1}{2}} \mathcal{E}^{*}(g, s ; r, n)
$$

(iii) If $0<s_{0}<\rho_{n}$, then

$$
\operatorname{Res}_{s=s_{0}} E^{*}\left(g, s, \Phi^{0}\right)=c(r, n) \operatorname{Res}_{s=\frac{r-1}{2}}\left(\left(s-\frac{r-1}{2}\right) \mathcal{E}^{*}(g, s ; r, n)\right) .
$$


Here the constant $c(r, n)$ is defined by $c(1,1)=1$ and

$$
c(r, 2 r-1)^{-1}=\frac{1}{2} \kappa L(3) L(5) \ldots L\left(2\left[\frac{r}{2}\right]-1\right),
$$

for $r>1$. If $1 \leq k \leq r-1$, then

Finally, for $\ell \geq 1$

$$
c(r, 2 r-1-k)^{-1}=c(r, 2 r-1)^{-1} 2 L(2) L(4) \ldots L\left(2\left[\frac{k}{2}\right]\right) .
$$

$$
c(r, 2 r-1+\ell)=-c(r, 2 r-1) \frac{1}{2} \kappa L(3) L(5) \ldots L\left(2\left[\frac{\ell-1}{2}\right]+1\right) .
$$

Theorem, [17]. (spherical second term identity) Let $s_{0}=r-\rho_{n}=r-\frac{n+1}{2}=\frac{k}{2}$ for $k \in$ $\mathbb{Z}>0$. Note that then $\mathcal{E}^{*}(g, s ; r, n)$ is associated to $O(r, r)=O\left(V_{r, r}\right)$ while $\mathcal{E}^{*}(g, s ; r-$ $k, n)$ is associated to the complementary space $O(r-k, r-k)=O\left(V_{r-k, r-k}\right)$. Let

$$
D(g, s ; r, n)=\mathcal{E}^{*}(g, s ; r, n)+\beta(s ; r, n) \gamma(s ; r, n) \mathcal{E}^{*}\left(g, s-\frac{k}{2} ; r-k, n\right)
$$

where

$$
\beta(s ; r, n)= \begin{cases}L(2 s) L(2 s-2) \ldots L\left(2 s-2\left[\frac{k-1}{2}\right]\right) & \text { if } r \text { is even } \\ L(2 s-1) L(2 s-3) \ldots L\left(2 s-2\left[\frac{k}{2}\right]+1\right) & \text { if } r \text { is odd }\end{cases}
$$

and with $\gamma(s ; r, n)$ defined inductively by $\gamma(s ; r, 2 r-1)=1$ and

$$
\gamma(s ; r, n)=\gamma\left(s-\frac{1}{2} ; r-1, n-1\right) \begin{cases}L\left(s-\frac{r-1}{2}-k+1\right) & \text { if } n \text { is even } \\ L\left(s-\frac{r-1}{2}+k\right) & \text { if } n \text { is odd. }\end{cases}
$$

Then $D(g, s ; r, n)$ has at most a simple pole at $s=\frac{r-1}{2}$ and, writing

$$
E^{*}\left(g, s, \Phi^{0}\right)=\frac{A_{-1}\left(g, \Phi^{0}\right)}{s-s_{0}}+A_{0}\left(g, \Phi^{0}\right)+O\left(s-s_{0}\right)
$$

we have

$$
A_{0}\left(g, \Phi^{0}\right)=c(r, n) \operatorname{Res}_{s=\frac{r-1}{2}} D(g, s ; r, n),
$$

where $c(r, n)$ is as in the previous Theorem.

REMARK: The main idea here is that $\mathcal{E}^{*}(g, s ; r, n)$ has a Laurent expansion (III.3.3), with a second order term which generates a copy of $\Pi_{n}\left(V_{r-k, r-k}\right)$ in $\mathcal{A}(G)$, while $\mathcal{E}^{*}(g, s ; r-$ $k, n)$ has only a simple pole at $s=\frac{r-k-1}{2}$, whose residue also generates a copy of $\Pi_{n}\left(V_{r-k, r-k}\right)$. The factor $\beta(s ; r, n) \gamma(s ; r, n)$ also has a simple pole at $s=\frac{r-1}{2}$, and has been taken so that the second order terms cancel in the sum $D(g, s ; r, n)$. Miraculously, the given choice exactly expresses the second term in the Laurent expansion of the Siegel Eisenstein series. Details of the proof will appear elsewhere.

Much work remains to be done to establish a second term identity in the general case. 


\section{IV.1. Applications to poles of Langlands $L$-functions.}

The rather complete information given in the previous sections about the poles of the Siegel Eisenstein series yields corresponding information about the poles of the standard Langlands $L$-functions for automorphic representations of the symplectic group.

First recall that the $L$-group of $G=S p(n)$ is ${ }^{L} G=S O(2 n+1, \mathbb{C}) \times W_{F}$ where $W_{F}$ is the global Weil group of $F$. Let $r:{ }^{L} G \longrightarrow G L(2 n+1, \mathbb{C})$ be the representation which is the standard representation on $\mathbb{C}^{2 n+1}$ on the first factor and is trivial on $W_{F}$. If $\pi \simeq \otimes_{v} \pi_{v}$ is an irreducible cuspidal automorphic representation of $G(\mathrm{~A})$, then for all places $v$ outside of a finite set $S=S(\pi)$, which contains all of the archimedean places, the local component $\pi_{v}$ of $\pi$ is the spherical constituent of an unramified principal series representation. Such an unramified principal series representation is determined by its Satake parameter $t_{v} \in{ }^{L} G$.

More precisely, let

$$
B=\{n m(a) \in P \mid \text { such that } a \text { is upper triangular }\}
$$

and write $B=T U$ where $U$ is the unipotent radical and

$$
T=\{m(a) \mid a=\text { diagonal }\}
$$

For any any non-archimedean place $v$ of $F$ and for any

$$
t_{v}^{0}=\operatorname{diag}\left(q_{v}^{-\lambda_{1}}, \ldots, q_{v}^{-\lambda_{n}}, 1, q_{v}^{\lambda_{1}}, \ldots, q_{v}^{\lambda_{n}}\right) \in S O(2 n+1, \mathbb{C})
$$

where $\lambda_{j} \in \mathbb{C}$, the induced representation $\operatorname{Ind}_{B_{v}}^{G_{v}}(\lambda)$ is given by the right multiplication action of $G_{v}$ on the space of smooth functions $f$ on $G_{v}$ such that

$$
f(u m(a) g)=\left|a_{1}\right|_{v}^{\lambda_{1}+n}\left|a_{2}\right|_{v}^{\lambda_{2}+n-1} \ldots\left|a_{n}\right|_{v}^{\lambda_{n}+1} f(g)
$$

where $a=\operatorname{diag}\left(a_{1}, \ldots, a_{n}\right) \in G L\left(n, F_{v}\right)$. Since $G_{v}=B_{v} K_{v}$ this representation has a unique $K_{v}$ invariant vector $f_{v}^{0}$ determined by the condition $f_{v}^{0}(k)=1$ for all $k \in K_{v}$. The the spherical constituent of $\operatorname{Ind}_{B_{v}}^{G_{v}}(\lambda)$ is the unique irreducible constituent containing $f_{v}^{0}$. Let

$$
t_{v}=t_{v}^{0} \times F r_{v} \in{ }^{L} G_{v}=S O(2 n+1, \mathbb{C}) \times W_{F_{v}}
$$


where $F r_{v}$ is a Frobenius element of $W_{F_{v}}$.

The local Euler factor attached to $\pi_{v}$ is then

$$
L_{v}\left(s, \pi_{v}, r\right)=\operatorname{det}\left(1-q_{v}^{-s} r\left(t_{v}\right)\right)^{-1}
$$

Note that it has degree $2 n+1$. The standard Langlands $L$-function of $\pi$ is the

$$
L^{S}(s, \pi, r)=\prod_{v \notin S} L_{v}\left(s, \pi_{v}, r\right)
$$

A little more generally, if $\chi$ is a character of $F_{\mathbf{A}}^{\times} / F^{\times}$, let $S=S(\pi, \chi)$ be the union of $S(\pi)$ with the set of non-archimedean places $v$ at which $\chi$ is ramified. Then, for $v \notin S$, define the twisted Euler factor

$$
L_{v}\left(s, \pi_{v}, \chi_{v}, r\right)=\operatorname{det}\left(1-q_{v}^{-s} \chi_{v}\left(\varpi_{v}\right) r\left(t_{v}\right)\right)^{-1}
$$

with $\varpi_{v}$ a generator of the maximal ideal in the ring of integers $\mathcal{O}_{v}$ of $F_{v}$, and define the $L$-function

$$
L^{S}(s, \pi, \chi, r)=\prod_{v \notin S} L_{v}\left(s, \pi_{v}, \chi_{v}, r\right)
$$

The meromorphic analytic continuation and functional equation of $L^{S}(s, \pi, \chi, r)$ can be obtained by the 'doubling method' integral representation of Piatetski-Shapiro and Rallis, $[\mathbf{3 0}, 31]$ and also $[\mathbf{4}, 8]$, which we now briefly recall. Let $\tilde{G}=S p(2 n)$ and let $\iota_{0}$ be the embedding $\iota_{0}: G \times G \longrightarrow \tilde{G}$ given by

$$
\iota_{0}\left(g_{1}, g_{2}\right)=\left(\begin{array}{llll}
a_{1} & & b_{1} & \\
& a_{2} & & b_{2} \\
c_{1} & & d_{1} & \\
& c_{2} & & d_{2}
\end{array}\right)
$$

where $g_{i}=\left(\begin{array}{cc}a_{i} & b_{i} \\ c_{i} & d_{i}\end{array}\right) \in G$. For $g \in G$, let

$$
g^{\vee}=\left(\begin{array}{ll}
1_{n} & \\
& -1_{n}
\end{array}\right) g\left(\begin{array}{ll}
1_{n} & \\
& -1_{n}
\end{array}\right)
$$

and let

$$
\iota\left(g_{1}, g_{2}\right)=\iota_{0}\left(g_{1}, g_{2}^{\vee}\right)
$$


Also let

$$
\delta=\left(\begin{array}{cccc}
0 & 0 & -\frac{1}{2} & \frac{1}{2} \\
\frac{1}{2} & \frac{1}{2} & 0 & 0 \\
1 & -1 & 0 & 0 \\
0 & 0 & 1 & 1
\end{array}\right) \in \tilde{G}
$$

Choose $f_{1}$ and $f_{2} \in \pi$ such that $f_{i}$ is invariant under $K_{v}$ for all $v \notin S$. Also choose $\Phi(s)=\otimes_{v} \Phi_{v}(s) \in I_{2 n}(s, \chi)$ such that $\Phi_{v}(s)=\Phi_{v}^{0}(s)$, the normalized $\tilde{K}_{v}=S p\left(2 n, \mathcal{O}_{v}\right)$ invariant vector in $I_{2 n, v}\left(s, \chi_{v}\right)$ for all $v \notin S$. Let $E^{*}(g, s, \Phi)$ be the normalized Siegel Eisenstein series on $\tilde{G}($ A) and consider the integral

(IV.1.14)

$$
Z^{*}\left(s, f_{1}, f_{2}, \Phi\right):=\int_{(G(F) \backslash G(\mathrm{~A})) \times(G(F) \backslash G(\mathrm{~A}))} f_{1}\left(g_{1}\right) \overline{f_{2}\left(g_{2}\right)} E^{*}\left(\iota\left(g_{1}, g_{2}\right), s \Phi\right) d g_{1} d g_{2} .
$$

The main identity of the doubling method [30] then asserts that

$$
Z^{*}\left(s, f_{1}, f_{2}, \Phi\right)=L^{S}\left(s+\frac{1}{2}, \pi, \chi, r\right)<\pi_{S}(\Phi(s)) f_{1}, f_{2}>
$$

where $\Phi_{S}(s)=\otimes_{v \in S} \Phi_{v}(s)$ is a function on $G_{S}=\operatorname{prod}_{v \in S} G_{v}$, and

$$
<\pi_{S}(\Phi(s)) f_{1}, f_{2}>=\int_{G_{S}}<\pi(g) f_{1}, f_{2}>\Phi_{S}(\delta \cdot \iota(g, 1), s) d g
$$

Note that the shift to $s+\frac{1}{2}$ in the $L$-function corresponds to the fact tha our Eisenstein series have $s=0$ as their center of symmetry while the Langlands $L$-functions have functional equations relating $s$ and $1-s$. ¿From (IV.1.14) it is clear that the poles of $Z^{*}\left(s, f_{1}, f_{2}, \Phi\right)$ areis from the poles of $E^{*}(g, s, \Phi)$. We can thus apply our previous results, noting that the Eisenstein series in question is on $\tilde{G}(\mathbb{A})$ rather than on $G(\mathbb{A})$.

We normalize $\chi$ by fixing an isomorphism $F_{\mathrm{A}}^{\times} / F^{\times} \simeq F_{\mathrm{A}}^{1} / F^{\times} \times \mathbb{R}_{+}^{\times}$and assuming that $\chi$ is trivial on the $\mathbb{R}_{+}^{\times}$factor. Then we have a precise description of the location of the poles of $L^{S}(s, \pi, \chi, r)$.

Theorem, [23].

(i) If $\chi^{2} \neq 1$, then $L^{S}(s, \pi, \chi, r)$ is entire.

(ii) If $\chi^{2}=1$, then $L^{S}(s, \pi, \chi, r)$ has at most simple poles, and these can only occur at the points $s \in\left\{1,2, \ldots,\left[\frac{n}{2}\right]+1\right\}$. 
Next we interpret the poles which occur. Let $V$ be a quadratic spce over $F$ of dimension $m$ and character $\chi_{V}$. Let $H=O(V)$ as before and, for an irreducible automorphic cuspidal representation $\pi$ of $G(\mathrm{~A})$, as above, let $\Theta(\pi)$ denote the space of automorphic forms on $H(\mathrm{~A})$ given by the theta integrals

$$
\theta(h ; f, \varphi)=\int_{G(F) \backslash G(\mathbf{A})} f(g) \theta(g, h ; \varphi) d g
$$

where $f \in \pi \subset \mathcal{A}(G)$ and $\varphi \in S\left(V(\mathrm{~A})^{n}\right)$ is $K$-finite and $K_{H}$-finite. Here $K_{H}$ is some fixed maximal compact subgroup of $H(\mathrm{~A})$.

Theorem, [23]. Let $\pi$ and $\chi$ be as before, with $\chi^{2}=1$. Suppose that $L^{S}(s, \pi, \chi, r)$ has a pole at the point $s=s_{0} \in\left\{1,2, \ldots,\left[\frac{n}{2}\right]+1\right\}$. Let $m=2 n+2-2 s_{0}$. Then there exists a quadratic space $V$ over $F$ with $\operatorname{dim}_{F} V=m$ and $\chi_{V}=\chi$ such that $\Theta_{V}(\pi) \neq 0$.

Thus the existence of a pole of $L^{S}(s, \pi, \chi, r)$ at $s_{0}$ indicates the non-triviality of a theta lift of $\pi$. The further to the right the pole occurs, the smaller the space $V$. For example, if $n$ is even, the rightmost possible pole occurs at $s_{0}=\frac{n}{2}+1$, and, if such a pole does occur, then $\pi$ has a non-trivial theta lift $\Theta_{V}(\pi)$ for some space $V$ of dimension $n$. This result was proved earlier by Piatetski-Shapiro and Rallis [32], using Andrianov's method [1,2]. By a result of $\mathrm{Li}[26], \Theta_{V^{\prime}}(\pi)$ must be zero for all quadratic spaces $V^{\prime}$ of dimension less than $n$.

The proof of this last result is based on the following:

Proposition, [23]. Let $V$ be a quadratic space over $F$ of dimension $m$ with $m \leq 2 n$.

(i) Suppose that $V$ is anisotropic and let $\bar{I}: \Pi_{2 n}(V) \longrightarrow \mathcal{A}(\tilde{G})$ be the intertwining map given by (II.2.4). Then, for $\varphi=\varphi_{1} \otimes \bar{\varphi}_{2} \in S\left(V(\mathrm{~A})^{2 n}\right)$,

$$
\begin{array}{rl}
\int_{[G \times G]} f_{1}\left(g_{1}\right) \overline{f_{2}\left(g_{2}\right)} \bar{I}\left(\iota\left(g_{1}, g_{2}\right), \varphi\right) d g_{1} & d g_{2} \\
& =\int_{H(F) \backslash H(\mathbf{A})} \theta\left(h ; f_{1}, \varphi_{1}\right) \overline{\theta\left(h ; f_{2} \varphi_{2}\right)} d h .
\end{array}
$$

Here $[G \times G]=(G(F) \backslash G(\mathrm{~A})) \times G(F) \backslash G(\mathrm{~A}))$.

(ii) Suppose that $V$ is isotropic and let $\bar{B}_{-1}: \Pi_{2 n}(V) \longrightarrow \mathcal{A}(\tilde{G})$ be the intertwining 
map given by (III.3.4). Then, for $\varphi=\varphi_{1} \otimes \bar{\varphi}_{2} \in S\left(V(\mathbb{A})^{2 n}\right)$,

$$
\begin{aligned}
& \int_{[G \times G]} f_{1}\left(g_{1}\right) \overline{f_{2}\left(g_{2}\right)} \bar{B}_{-1}\left(\iota\left(g_{1}, g_{2}\right), \varphi\right) d g_{1} d g_{2} \\
&=c \int_{H(F) \backslash H(\mathbf{A})}\left(\theta\left(h ; f_{1}, \varphi_{1}\right) \overline{\theta\left(h ; f_{2} \varphi_{2}\right)}\right) * z^{\prime} d h,
\end{aligned}
$$

where $z^{\prime} \in \mathfrak{z}\left(\mathfrak{h}_{v}\right)$ is the element, defined in the Theorem of section II.2, used to regularize the theta integral.

This result relates that residues of $Z^{*}\left(s, f_{1}, f_{2}, \Phi\right)$ to the (regularized) Petersson inner products of theta lifts $\theta(h ; f, \varphi)$. This relation - Rallis' inner product formula, [34] - was the starting point of the work of Piatetski-Shapiro and Rallis on the 'doubling method'.

\section{IV.2 Translation to classical language.}

It might be useful to explain the relation between the adelic Eisenstein series $E(g, s, \Phi)$ on $G(\mathrm{~A})$ and the classical Eisenstein series of Siegel, $[3 \mathbf{3 8}, 37,15,7,5]$. For this it is simplest to work over $F=\mathbb{Q}$.

Note that, by the strong approximation theorem,

$$
G(\mathbb{A})=G(\mathbb{Q}) G(\mathbb{R}) K^{\prime}
$$

where $K^{\prime}$ is any compact open subgroup of $G\left(\mathbb{A}_{f}\right)$. We will usually take $K^{\prime}$ to be a subgroup of finite index in $K=\prod_{p} K_{p}$ where $K_{p}=S p\left(n, \mathbb{Z}_{p}\right)$. Note that we are slightly changing the notation of the previous sections. Let

$$
\Gamma=G(\mathbb{Q}) \cap(G(\mathbb{R}) K)=S p(n, \mathbb{Z})
$$

and let

$$
\Gamma=G(\mathbb{Q}) \cap\left(G(\mathbb{R}) K^{\prime}\right) .
$$

Let

$$
\Phi(s)=\Phi_{\infty}(s) \otimes \Phi_{f}(s)
$$

with

$$
\Phi_{f}(s)=\otimes_{p} \Phi_{p}(s)
$$


be a standard (restriction to $K$ is independent of $s$ ) factorizable section. We assume that $\Phi_{f}(s)$, the finite part of $\Phi(s)$ is invariant under $K^{\prime}$ ( as it must be for a sufficiently small $\left.K^{\prime}\right)$; and thus the Eisenstein series $E(g, s, \Phi)$, which is left $G(\mathbb{Q})$-invariant and right $K^{\prime}$-invariant, is determined by its restriction to $G(\mathbb{R})$, which we view as embedded in $G(\mathbb{A})$ via $g_{\infty} \mapsto\left(g_{\infty}, 1, \ldots\right)$.

Note that

$$
P(\mathbb{Q}) \backslash G(\mathbb{Q}) \simeq(P(\mathbb{Q}) \cap \Gamma) \backslash \Gamma
$$

Thus, we have

$$
E\left(g_{\infty}, s, \Phi\right)=\sum_{\gamma \in P(\mathbb{Q}) \cap \Gamma} \Phi_{\infty}\left(\gamma g_{\infty}\right) \Phi_{f}(\gamma)
$$

so that we must determine the two factors $\Phi_{f}(\gamma)$ and the function $\Phi_{\infty}\left(\gamma g_{\infty}\right)$. Note that the function $\Phi_{f}(\gamma, s)$ depends only on the restriction of $\Phi_{f}(s)$ to $K$, and that the data $\Phi_{f}(s)$ and $\Phi_{\infty}(s)$ can be varied independently.

Assume that $N \in \mathbb{Z}_{>0}$ is such that $K^{\prime}=\prod_{p \mid N} K_{p}^{\prime} \times \prod_{p \nmid N} K_{p}$ and that $\Phi_{p}(s)=$ $\Phi_{p}^{0}(s)$, the normalized spherical section of (II.1.10), for all $p$ prime to $N$. Then

$$
\Phi_{f}(\gamma)=\prod_{p \mid N} \Phi_{p}(\gamma)
$$

Here the functions $\phi_{p}=\Phi_{p}: K^{\prime} \backslash K \longrightarrow \mathbb{C}$ can be chosen arbitrarily. Non-trivial examples in the case $n=3$ can be found in section 3 of [9].

Recall that $G_{\infty}=S p(n, \mathbb{R})=P(\mathbb{R}) K_{\infty}$ where $K_{\infty} \simeq U(n)$ as in section I.1. In particular, $\Phi_{\infty}(s)$ is determined by its restriction to $K_{\infty}$. The simplest possible choice of $\Phi_{\infty}(s)$ will be the function determined by

$$
\Phi_{\infty}^{\ell}(k, s)=(\operatorname{det} \mathbf{k})^{\ell}
$$

where $\mathbf{k} \in U(n)$ corresponds to $k \in S p(n, \mathbb{R})$. For this choice of $\Phi_{\infty}(s)$, the function $g_{\infty} \mapsto E\left(g_{\infty}, s, \Phi\right)$ is an eigenfunction for the right action of $K_{\infty}$, so it suffices to describe its values on $P(\mathbb{R})$. Write $g=n(x) m(v) \in P(\mathbb{R})$, and set

$$
z=x+i y=x+i^{t} v v=g\left(i \cdot 1_{n}\right) \in \mathfrak{H}_{n}
$$


the Siegel space of genus $n$. Let $\gamma=\left(\begin{array}{ll}a & b \\ c & d\end{array}\right)$, and let $\gamma g=n m(\alpha) k$ for the Iwasawa decomposition, with $\alpha \in G L^{+}(n, \mathbb{R})$, i.e., with $\operatorname{det} \alpha>0$. Then we have

$$
\left(0,1_{n}\right) \cdot \gamma g\left(\begin{array}{c}
1_{n} \\
i \cdot 1_{n}
\end{array}\right)=i \cdot{ }^{t} \alpha^{-1} \mathbf{k}
$$

On the other hand,

$$
\left(0,1_{n}\right) \cdot \gamma g\left(\begin{array}{c}
1_{n} \\
i \cdot 1_{n}
\end{array}\right)=i \cdot(c \bar{z}+d)^{t} v^{-1}
$$

so that

$$
\operatorname{det}(\alpha)=\operatorname{det}(v) \cdot|\operatorname{det}(c z+d)|^{-1}
$$

and

$$
\operatorname{det}(\mathbf{k})=\frac{\operatorname{det}(c \bar{z}+d)}{|\operatorname{det}(c z+d)|}
$$

This yields:

$$
\begin{aligned}
\Phi_{\infty}^{\ell}(\gamma g, s) & =\operatorname{det}(v)^{s+\rho_{n}}|\operatorname{det}(c z+d)|^{-s-\rho_{n}-\ell} \operatorname{det}(c \bar{z}+d)^{\ell} \\
& =\operatorname{det}(y)^{\frac{1}{2}\left(s+\rho_{n}\right)} \operatorname{det}(c z+d)^{-\frac{1}{2}\left(s+\rho_{n}+\ell\right)} \operatorname{det}(c \bar{z}+d)^{-\frac{1}{2}\left(s+\rho_{n}-\ell\right)} \\
& =\operatorname{det}(y)^{\frac{1}{2}\left(s+\rho_{n}\right)} \operatorname{det}(c z+d)^{-\ell}|\operatorname{det}(c z+d)|^{-s-\rho_{n}+\ell} .
\end{aligned}
$$

More generally, since $\Phi_{\infty}(s)$ is standard and $K_{\infty}$-finite, we may write

$$
\Phi_{\infty}(k, s)=\phi(\mathbf{k})
$$

for smooth function $\phi$ on $U(n)$ which is left invariant under $S O(n) \subset U(n)$. Then, taking $g$ and $z$ as in (IV.2.9),

$$
\Phi_{\infty}(\gamma g, s)=\operatorname{det}(v)^{s+\rho_{n}} \cdot|\operatorname{det}(c z+d)|^{-s-\rho_{n}} \cdot \phi(\mathbf{k})
$$

where $\mathbf{k}=\mathbf{k}(\gamma, z)$ is as in (IV.2.11). To find $\mathbf{k}(\gamma, z)$ more explicitly, observe that if we set

$$
X=i^{t} \alpha^{-1} \mathbf{k}=i \cdot(c \bar{z}+d)^{t} v^{-1}
$$


as in (IV.2.12), then

$$
\bar{X}^{-1}=i^{t} \mathbf{k}^{t} \alpha
$$

Thus

$$
\begin{aligned}
\bar{X}^{-1} X & =-{ }^{t} \mathbf{k} \cdot \mathbf{k} \\
& =-{ }^{t} v(c z+d)^{-1}(c \bar{z}+d)^{t} v^{-1}
\end{aligned}
$$

and so

$$
{ }^{t} \mathbf{k k}={ }^{t} v(c z+d)^{-1}(c \bar{z}+d)^{t} v^{-1}
$$

Note that, by left $S O(n)$ invariance, the function $\phi$ depends only on ${ }^{t} \mathbf{k k}$ and on $\operatorname{det}(\mathbf{k})$. Thus, in general,

$$
E(g, s, \Phi)=\operatorname{det}(y)^{\frac{1}{2}\left(s+\rho_{n}\right)} \sum_{\gamma \in \Gamma_{\infty} \backslash \Gamma}|\operatorname{det}(c z+d)|^{-s-\rho_{n}} \phi_{\infty}(\mathbf{k}(\gamma, z)) \prod_{p \mid N} \phi_{p}(\gamma)
$$

We will not continue the discussion of the general case.

Suppose, now, that $N=1$. Note that the character $\chi$ must be everywhere unramified and hence trivial, since $F=\mathbb{Q}$. Then, for $g$ and $z$ related by (IV.2.10) and for $\Phi_{\infty}(s)=$ $\Phi_{\infty}^{\ell}(s)$, we have

$$
E(g, s, \Phi)=\operatorname{det}(y)^{\ell} \sum_{\Gamma_{\infty} \backslash \Gamma} \operatorname{det}(c z+d)^{-\ell}|\operatorname{det}(c z+d)|^{-s-\rho_{n}+\ell} \operatorname{det}(y)^{\frac{1}{2}\left(s+\rho_{n}-\ell\right)}
$$

$(\mathrm{IV} .2 .23)$

$$
=\operatorname{det}(y)^{\ell} E_{\text {Classical }}\left(z, s+\rho_{n}-\ell\right) \text {. }
$$

Here

$$
E_{\text {Classical }}(z, s)=\sum_{(c, d)} \operatorname{det}(c z+d)^{-\ell}|\operatorname{det}(c z+d)|^{-s}
$$

is the most classical Siegel Eisenstein series of weight $\ell$.

It is amusing to determine when this level 1 (i.e., $N=1$ ) Eisenstein series is incoherent, say at the point $s=0$. We assume that $n>1$ is odd and set $m=n+1=2 r$. 
Recall that $\chi=1$. Also recall that, in the non-archimedean case, the local (unitarizable) induced representations decompose as:

$$
I_{n, p}(0)=R_{n, p}\left(V_{r, r}\right) \oplus R_{n, p}\left(V_{B}\right)
$$

where $V_{r, r}$ is the split space and $V_{B}$ is the 'quaternionic, space (i.e., the direct sum of the norm form from the quaternion algebra $B=B_{p}$ over $\mathbb{Q}_{p}$ and $V_{r-2, r-2}$. In the archimedean case:

(IV.2.26) $\quad I_{n, \infty}(0)=R_{n}(m, 0) \oplus R_{n}(m-2,2) \oplus \cdots \oplus R_{n}(r, r) \oplus \cdots \oplus R_{n}(0, m)$,

if $m \equiv 0 \bmod 4$, and

$(\mathrm{IV} .2 .27) \quad I_{n, \infty}(0)=R_{n}(m-1,1) \oplus R_{n}(m-3,3) \oplus \cdots \oplus R_{n}(r, r) \oplus \cdots \oplus R_{n}(1, m-1)$

if $m \equiv 2 \bmod 4$.

Note that $R_{n, p}\left(V_{r, r}\right)$ contains the spherical vector $\Phi_{p}^{0}(0)$, so that our local components lie in this subspace for every finite place. Since the Hasse invariant of $V_{r, r}$ is

$$
\epsilon_{p}\left(V_{r, r}\right)=(-1,-1)_{p}^{\frac{r(r-1)}{2}}
$$

we see that our global section will be incoherent precisely when $\Phi_{\infty}^{\ell}(0)$ lies in some $R_{n}(p, q)$ for which

$$
\epsilon_{\infty}\left(V_{p, q}\right)=(-1)^{\frac{q(q-1)}{2}}=-\epsilon_{\infty}\left(V_{r, r}\right)=-(-1)^{\frac{r(r-1)}{2}}
$$

Note that the condition $\chi_{\infty}=1$ implies that $\ell$ is even. In the range

$$
-r \leq \ell \leq r \quad \ell \text { even, }
$$

the vector

$$
\Phi_{\infty}^{\ell}(0) \in R_{n}(r+\ell, r-\ell)
$$

Thus, in this range, (IV.2.22) becomes

$$
(-1)^{\frac{(r-\ell)(r-\ell-1)}{2}}=-(-1)^{\frac{r(r-1)}{2}}
$$


i.e., simply $\ell \equiv 2 \bmod 4$, since $\ell$ is even. On the other hand, if $\ell \geq m$ (resp. $\ell \leq-m$ ), then

$$
\Phi_{\infty}^{\ell}(0) \in \begin{cases}R_{n}(m, 0) \quad\left(\operatorname{resp} . R_{n}(0, m)\right) & \text { if } m \equiv 0 \bmod 4 \\ R_{n}(m-1,1) \quad\left(\operatorname{resp} . R_{n}(1, m-1)\right) & \text { if } m \equiv 2 \bmod 4\end{cases}
$$

Proposition. Consider the level 1 Eisenstein series $E\left(g, s, \Phi^{\ell}\right)$ of (even) weight $\ell$ at $s=0$.

(i) If $-r \leq \ell \leq r$, then $E\left(g, s, \Phi^{\ell}\right)$ is coherent if $\ell \equiv 0 \bmod 4$ and incoherent if $\ell \equiv 2 \bmod 4$.

(ii) If $\ell \geq r$, then $E\left(g, s, \Phi^{\ell}\right)$ is coherent if $r \equiv 0,1 \bmod 4$ and incoherent if $r \equiv 2,3 \bmod 4$.

(iii) If $\ell \leq-r$, then $E\left(g, s, \Phi^{\ell}\right)$ is coherent if $r \equiv 0,3 \bmod 4$ and incoherent if $r \equiv 1,2 \bmod 4$.

In particular, assume that $m=n+1 \equiv 0 \bmod 4$, and take $\ell=r=\frac{n+1}{2}$, so that $\Phi_{\infty}^{\ell}(0) \in R_{n}(m, 0)$. If $n \equiv 7 \bmod 8$, then $\ell \equiv 0 \bmod 4$ and $E\left(g, s, \Phi^{\ell}\right)$ is coherent at $s=0$. Its value $E\left(g, 0, \Phi^{\ell}\right)$ is a non-zero holomorphic Siegel Eisenstein series which is expressible as the usual average over classes in a genus of positive definite quadratic forms of dimension $m=n+1$ and character $\chi_{V}=1$. On the other hand, if $n \equiv 3 \bmod 8$, then $\ell \equiv 2 \bmod 4$ and $E\left(g, s, \Phi^{\ell}\right)$ is incoherent at $s=0$. The explicit calculation of $E^{\prime}\left(g, 0, \Phi^{\ell}\right)$ is then of interest! The case $n=3$, and its generalizations to arbitrary $N$ play a key role in $[\mathbf{9}, 12]$ and the generalization of [10] mentioned in $\S 12$ of [9].

As another example of an incoherent Eisenstein series, suppose that $n=2, N=1$ (so $\chi=1$ ), and $\ell=2$, so that we are considering the level 1 Eisenstein series of weight 2 and genus 2 . In this case, we consider the point $s_{0}=2-\frac{3}{2}=\frac{1}{2}$. Then,

$$
I_{2, p}\left(\frac{1}{2}\right)=R_{2, p}\left(V_{2,2}\right)+R_{2, p}\left(V_{B}\right)
$$

where the sum is no longer direct. However $\Phi_{p}^{0}\left(\frac{1}{2}\right)$ lies in $R_{2, p}\left(V_{2,2}\right)$ and not in $R_{2, p}\left(V_{B}\right)$. The product of the non-archimedean Hasse invariants of the spaces $V_{2,2}$ must equal the archimedean Hasse invariant $\epsilon_{\infty}\left(V_{2,2}\right)=(-1,-1)_{\mathbb{R}}=-1$. On the other hand,

$$
I_{2, \infty}\left(\frac{1}{2}\right)=R_{2, \infty}(2,2) \supsetneqq R_{2, \infty}(4,0)+R_{2, \infty}(0,4),
$$

and the vector $\Phi_{\infty}^{2}\left(\frac{1}{2}\right)$ lies in the submodule $R_{2, \infty}(4,0)$. The global section is not literally incoherent in the sense of section II.1, since it lies in the space $\Pi_{2}\left(V_{2,2}\right)$. However, the 
complementary space $V_{0}$ to $V_{2,2}$ is just the split binary space $V_{1,1}$, and our section $\Phi^{2}\left(\frac{1}{2}\right)$ lies in the kernel of the map $\Pi_{2}\left(V_{2,2}\right) \longrightarrow \Pi_{2}\left(V_{1,1}\right)$ of (III.1.7). Thus, by (ii) of the second Theorem of section III.1, $E\left(g, s, \Phi^{2}\right)$ is holomorphic at $s=\frac{1}{2}$. The same remarks apply if we take any $\Phi_{\infty}\left(\frac{1}{2}\right) \in R_{2, \infty}(4,0)$, so that there is a map

$$
\begin{aligned}
\Pi_{2}(\mathcal{C})=R_{2, \infty}(4,0) \otimes\left(\otimes_{p} R_{2, p}\left(V_{2,2}\right)\right) & \longrightarrow \mathcal{A}(G) \\
\Phi & \mapsto E\left(g, \frac{1}{2}, \Phi\right) .
\end{aligned}
$$

This map is only intertwining modulo the image of $\bar{A}_{-1}: \Pi_{2}\left(V_{1,1}\right) \longrightarrow \mathcal{A}(G)$, and thus we obtain an extension

$$
0 \longrightarrow \Pi_{2}\left(V_{1,1}\right) \longrightarrow Y \longrightarrow \Pi_{2}(\mathcal{C}) \longrightarrow 0
$$

where $Y$ is the inverse image in $\mathcal{A}(G)$ of the image of $\Pi_{2}(\mathcal{C})$ in $\mathcal{A}(G) / \operatorname{Im}\left(\bar{A}_{-1}\right)$. Note that the vector $\Phi_{\infty}^{2}\left(\frac{1}{2}\right) \in R_{2, \infty}(4,0)$ is 'holomorphic', i.e., is killed by the $\bar{\partial}$ operator:

$$
\bar{\partial} f=\sum_{i} X_{i} f \cdot \omega_{i}
$$

where the sum is over a basis $X_{i}$ for $\mathfrak{p}_{-} \subset \mathfrak{g}$, the antiholomorphic tangent space to $\mathfrak{H}_{2}$ at $i \cdot 1_{2}$, and $\omega_{i}$ runs over the dual basis for $\mathfrak{p}_{-}^{*}$. Since $E\left(g, \frac{1}{2}, \Phi^{2}\right) \in Y$, we conclude that

$$
\bar{\partial} E\left(g, \frac{1}{2}, \Phi^{2}\right) \in \Pi_{2}\left(V_{1,1}\right) \otimes \mathfrak{p}_{-}^{*} .
$$

In fact, the function $E\left(g, \frac{1}{2}, \Phi^{2}\right)$ has recently been written out explicitly as a Fourier series by Kohnen [16] and also by Nagaoka [29]. It would be of interest to give an explicit expression for the binary 'theta integral' (cf. section 3.1 of [24]) $\bar{\partial} E\left(g, \frac{1}{2}, \Phi^{2}\right)$.

This last example suggests that the definition of incoherent sections in section II.1 ought to be slightly extended in the range $n+1<m \leq 2 n$.

Similar non-trivial extensions

$$
0 \longrightarrow \operatorname{Im}\left(A_{0}\right) \longrightarrow Y \longrightarrow \Pi_{n}(\mathcal{C}) \longrightarrow 0
$$

can be constructed by considering the map

$$
\begin{array}{rcc}
E^{\prime}(0): \Pi_{n}(\mathcal{C}) & \longrightarrow & \mathcal{A}(G) \\
\| & & \downarrow \\
\overline{E^{\prime}(0)}: \Pi_{n}(\mathcal{C}) & \hookrightarrow & \mathcal{A}(G) / \operatorname{Im}\left(A_{0}\right)
\end{array}
$$

where $A_{0}$ is as in (III.1.10), and $Y$ is the inverse image in $\mathcal{A}(G)$ of the image of $\overline{E^{\prime}(0)}$. The non-triviality follows from the non-vanishing of $\overline{E^{\prime}(0)}$ together with (ii) of the last Theorem in section II.2. Perhaps such extensions have some motivic meaning? 


\section{REFERENCES}

1. A. Andrianov, The multiplicative arithmetic of Siegel modular forms, Russian Math. Surveys 34 (1979), 75-148.

2. A. Andrianov and V. L. Kalinin, On the analytic properties of standard zeta functions of Siegel modular forms, Math. USSR, Sbornik 35 (1979), 1-17.

3. J. Arthur, Eisenstein series and the trace formula, Proc. Symp. Pure Math. 33 (1979), 27-63.

4. S. Böcherer, Über die Funktionalgleichung automorpher L -Funktionen zur Siegelschen Modulgruppe, J. Reine Angew. Math. 362 (1985), 146-168.

5. S. Böcherer and R. Schulze-Pillot, Siegel modular forms and theta functions attached to quaternion algebras, Nagoya Math. J. 121 (1991), 35-96.

6. A. Deitmar and A. Kreig, Theta correspondence for Eisenstein series, Math. Zeit. 208 (1991), 273-288.

7. P. Feit, Poles and residues of Eisenstein series for symplectic and unitary groups, Memoirs of AMS 61 (1986).

8. P. Garrett, Pullbacks of Eisenstein series; applications, in "Automorphic Forms of Several Variables," Tanaguchi Symposium, Katata, 1983, Birkhäuser, Boston, 1984.

9. B. H. Gross and S. Kudla, Heights and the central critical values of triple product $L$-functions, Compositio Math. 81 (1992), 143-209.

10. B. H. Gross and D. Zagier, Heegner points and the derivatives of $L$-series, Inventiones math. 84 (1986).

11. R. Gustafson, The degenerate principal series of $S p(2 n)$, Memoirs of AMS. 248 (1981).

12. M. Harris and S. Kudla, The central critical value of a triple product $L$-function, Annals of Math. 133 (1991), 605-672.

13. R. Howe, $\theta$-series and invariant theory, Proc. Symp. Pure Math. 33 (1979), 275-285, in "Automorphic Forms, Representations and $L$-functions,", pp..

14. C. Jantzen, Degenerate principal series for symplectic groups, to appear in Mem. of the Amer. Math. Soc..

15. V. L. Kalinin, Eisenstein series on the symplectic group, Math. USSR, Sbornik 32 (1977), 449-476.

16. W. Kohnen, Class numbers, Jacobi forms and Siegel Eisenst ein series of weight 2 on $S p_{2}(\mathbb{Z})$, preprint (1991).

17: S. Kudla, unpublished notes.

18. S. Kudla and S. Rallis, On the Weil-Siegel formula, J. Reine Angew. Math. 387 (1988), 1-68.

19. S. Kudla and S. Rallis, On the Weil-Siegel formula II: the isotropic convergent case, J. Reine Angew. Math. 391 (1988), 65-84.

20. S. Kudla and S. Rallis, Degenerate principal series and invariant distributions, Israel Journal of Math. 69 (1990), 25-45.

21. S. Kudla and S. Rallis, Ramified degenerate principal series, to appear in Israel Math. J..

22. S. Kudla and S. Rallis, Poles of Eisenstein series and $L$-functions,, in "Festschrift in honor of I. Piatetski-Shapiro," Israel Mathematical Conference Proceedings, Weizmann Science Press of Israel, 1990, pp. 81-110. 
23. S. Kudla and S. Rallis, $\boldsymbol{A}$ regularized Siegel-Weil formula: the first term identity, preprint (1992).

24. S. Kudla, S. Rallis, and D. Soudry, On the degree $5 L$-function for $S p(2)$, Inventiones math. 107 (1992), 483-541.

25. R. P. Langlands, On the notion of an automorphic representation; Proc. Symp. Pure Math. 33 (1979), 203-207.

26. Jian-Shu Li, Non-existence of singular cusp forms, Compositio Math. 83 (1992), 43-51.

27. H. Maass, "Siegel's Modular Forms and Dirichlet Series," Lecture Notes in Math., Springer-Verlag, New York, 1971.

28. C. Moeglin, M.-F. Vigneras, and J.-L. Waldspurger, "Correspondence de Howe sur un corps p-adique," Lecture Notes in Math., Springer-Verlag, New York, 1987.

29. S. Nagaoka, $A$ note on the Siegel Eisenstein series of weight 2 on $S p_{2}(\mathbb{Z})$, preprint (1992).

30. I. I. Piatetski-Shapiro and S. Rallis, " $L$-functions for classical groups," Lecture Notes in Math., Springer-Verlag, New York, 1987, pp. 1-52.

31. I. I. Piatetski-Shapiro and S. Rallis, Rankin triple L-functions, Compositio Math. 64 (1987), 333-399.

32. I. I. Piatetski-Shapiro and S. Rallis, $\boldsymbol{A}$ new way to get Euler products, J. Reine Angew. Math. 392 (1988), 110-124.

33. S. Rallis, On the Howe duality conjecture, Compositio Math. 51 (1984), 333-399.

34. S. Rallis, Injectivity properties of liftings associated to Weil representations, Compositio Math. 52 (1984), 139-169.

35. S. Rallis, " $L$-functions and the Oscillator Representation," Lecture Notes in Math., Springer-Verlag, New York, 1987.

36. S. Rallis, Poles of standard $L$-functions, in "Proceedings of the International Congress of Mathematics, Kyoto," Springer-Verlag, New York, 1990, pp. 833-845.

37. G. Shimura, On Eisenstein series, Duke Math. J. 50 (1983), 417-476.

38. C. L. Siegel, "Gesammelte Abhandlungen I,II,III," Springer-Verlag, Berlin, Heidelberg, New York, 1966.

39. J.-L. Waldspurger, Demonstration d'une conjecture de duality de Howe dans le case p-adiques, $p \neq 2$, in "Festschrift in Honor of Piatetski-Shapiro," Israel Math. Conf. Proc., 1990, pp. 267-324.

40. A. Weil, Sur certains groupes d'opérateurs unitaires, Acta Math. 111 (1964), 143-211.

41. A. Weil, Sur la formule de Siegel dans la théorie des groupes classiques, Acta Math. 113 (1965), 1-87.

Department of Mathematics,

University of Maryland,

College Park, MD, 20742, U.S.A 